\title{
Investigating gravitational collapse of a pebble cloud to form transneptunian binaries
}

\author{
J. E. Robinson ${ }^{1}$, W. C. Fraser ${ }^{1,2}$, A. Fitzsimmons ${ }^{1}$, and P. Lacerda ${ }^{1}$ \\ ${ }^{1}$ Astrophysics Research Centre, Queen's University Belfast, University Road, BT7 1NN, Belfast, UK \\ e-mail: j.e.robinson@qub.ac.uk \\ ${ }^{2}$ Herzberg Astronomy and Astrophysics Research Centre, National Research Council of Canada, 5071 West Saanich Road, \\ Victoria BC V9E 2E7, Canada
}

Received 7 January 2020 / Accepted 8 August 2020

\begin{abstract}
Context. A large fraction of transneptunian objects are found in binary pairs, $\sim 30 \%$ in the cold classical population between $a_{\text {hel }} \sim 39$ and $\sim 48$ AU. Observationally, these binaries generally have components of similar size and colour. Previous work has shown that gravitational collapse of a pebble cloud is an efficient mechanism for producing such systems. Since the bi-lobate nature of $2014 \mathrm{MU}_{69}$ (Arrokoth) was discovered, interest in gravitational collapse as a pathway for forming contact binaries has also grown.

Aims. We investigate the formation of binary systems through gravitational collapse by considering a wider range of binary masses than previous studies. We analysed in detail the properties of the bound systems that are formed and compared them to observations. Methods. We performed $N$-body simulations of gravitational collapse of a pebble cloud using the REBOUND package, with an integrator designed for rotating reference frames and robust collision detection. We conducted a deep search for gravitationally bound particles at the end of the gravitational collapse phase and tested their stability. For all systems produced, not just for the most massive binaries, we investigated the population characteristics of their mass and orbital parameters.

Results. We found that gravitational collapse is an efficient producer of bound planetesimal systems. On average, about 1.5 bound systems were produced per cloud in the mass range studied here. In addition to large equal-sized binaries, we found that gravitational collapse produces massive bodies with small satellites and low-mass binaries with a high mass ratio. Our results disfavour the collapse of high-mass clouds, in line with reported upper mass limits of clouds formed by the streaming instability. Gravitational collapse can create binary systems analogous to Arrokoth, and collisions in a collapsing cloud should be gentle enough to preserve a bi-lobed structure.
\end{abstract}

Key words. Kuiper belt: general - minor planets, asteroids: general - planets and satellites: formation

\section{Introduction}

The transneptunian objects, also known as Kuiper belt objects, are the mainly icy Solar System bodies beyond the orbit of Neptune. They are generally classified by their heliocentric orbits according to the taxonomy laid out by Gladman et al. (2008). The main dynamical classes are cold classicals, hot classicals, resonant objects, scattered disc, and fossilized scattered disc. A classical object is a transneptunian object that is not under the influence of or has been scattered by Neptune. Most classicals are found on orbits between the 3:2 and 2:1 Neptune resonances. In this case, "cold" refers to objects on heliocentric orbits that are less dynamically excited (they have lower eccentricity and inclination) than the "hot" objects. The transneptunian objects contain some of the most primitive solid material in the Solar System, and their physical properties and dynamical structure provide a valuable insight into Solar System history. A particularly interesting property of the transneptunian populations is the fraction of bodies in mutual orbit binary pairs, which varies across the different dynamical classes. For example, unlike the excited objects of which only $\sim 10 \%$ are in binary pairs (Stephens \& Noll 2006), the predominantly red surface colour and dynamically quiescent cold classical objects exhibit a high binary fraction of $\sim 30 \%$ (Fraser et al. 2017; Grundy et al. 2011; Noll et al. 2008).
These transneptunian binaries (TNBs) generally have similar-size components (Noll et al. 2008), both of which have similar surface colours (Benecchi et al. 2009; Marsset et al. 2020). Furthermore, some TNBs have wide orbital separations relative to the mutual Hill radius of the binary $\left(a_{\text {bin }} / R_{\text {Hill }}>0.05\right.$, where $a_{\text {bin }}$ is the binary orbit semimajor axis and $R_{\text {Hill }}$ is the Hill radius of the binary system). These properties are rather unique, especially when compared to asteroidal binary systems, which generally have smaller secondaries on tighter orbits (Walsh \& Jacobson 2015). They also display some interesting distributions in the orientation of their binary orbits. The most recent set of full orbital solutions (Grundy et al. 2019) shows that the tight binaries with $a_{\text {bin }} / R_{\text {Hill }}<0.05$ have a majority of prograde orbits (22 out of 26), whereas the wide binaries have a somewhat higher fraction of retrograde systems (6 out of 9 are prograde). However, we note that assuming Poisson statistics, the number of data points for the wide systems is too low to definitively state that their retrograde fraction is different from that of the tight binaries. It is clear, however, that the wide systems tend not to be highly inclined relative to their heliocentric orbits compared to the tight systems, which exhibit a full range of inclinations (Parker et al. 2011; Grundy et al. 2011, 2019). These unique properties present the opportunity of providing excellent constraints for theories of planetesimal and binary formation, and 
the subsequent evolution of the small-body population from the early Solar System to the present day.

In this work we focus on the formation of binary systems that are representative of the TNBs. The current literature knows two main classes of formation mechanisms: hierarchical coagulation and gravitational instability. The first has many sub-mechanisms, but they involve in general two or more planetesimals interacting with some loss of energy, which results in two bodies remaining in a bound pair. Some proposed mechanisms for removing excess energy include the ejection of a third body $\left(\mathrm{L}^{3}\right)$, dynamical friction with background small bodies $\left(\mathrm{L}^{2} \mathrm{~s}\right)$ (Goldreich et al. 2002), or collisions (Weidenschilling 2002; Funato et al. 2004). These methods can explain some properties of the TNBs, but may either be too inefficient, require unrealistic protoplanetary disc conditions, or fail to reproduce all the observed binary properties, for instance same colour components or the distribution of the binary orbit inclination.

The second mechanism, gravitational instability, was originally proposed as a method of forming planetesimals that overcomes the various barriers that oppose the growth of protoplanetary disc dust grains into larger bodies, for example the bouncing, fragmentation, and radial-drift barriers (see Weidenschilling 1977; Brauer et al. 2008; Güttler et al. 2010; Birnstiel et al. 2016). In this mechanism, solid particles in the gaseous protoplanetary disc are concentrated by the streaming instability (Youdin \& Goodman 2005) until the mutual self-gravity of a cluster is high enough for the cluster to collapse. During this collapse the particles collide and merge, allowing the rapid growth of $\sim 100 \mathrm{~km}$ planetesimals whilst overcoming the barriers above (Johansen et al. 2007, 2015; Simon et al. 2016). This mechanism was first proposed as a possible route to TNB formation by Nesvorný (2008) and Nesvorný et al. (2010). The particle clumps formed by streaming instability generally have some rotation as a result of vorticity in the gaseous protoplanetary disc. This means that it is difficult to form a single object without removing angular momentum from the particle cloud. Instead it is more probable for the cloud to collapse and transfer its angular momentum into a binary planetesimal system. For a given binary orbit the angular momentum of the binary is maximised when the components have equal mass. The streaming instability has been shown to be robust across a range of protoplanetary disc conditions, generally requiring a solid-to-gas density 2-5 times that of the solar ratio, see Johansen et al. (2009), Yang et al. (2017) and also Carrera et al. (2017) and Nesvorný et al. (2019).

The gravitational collapse of pebble clouds formed by streaming instability is extremely efficient at forming binary systems with the unusual properties of the TNBs: similar-size components, wide binary orbits, and similar colours (as the bodies formed simultaneously from the same cloud of material). Moreover, the inclination of a binary formed by gravitational collapse of a pebble cloud is dependent on the inclination of the cloud from which it forms. The clouds formed by streaming instability have a distribution of prograde and retrograde rotations, which means that binary formation by gravitational collapse can reproduce the observed binary inclination distribution (Nesvorný et al. 2019).

Since the original work by Nesvorný et al. (2010), who proposed gravitational collapse as a binary formation mechanism of TNBs, there has been a significant amount of research that supports this theory. For example Fraser et al. (2017) investigated the population of blue surface colour objects amongst cold classicals, nearly all of which exist as dynamically fragile, widely separated binary pairs. Different surface colours imply a different composition and therefore a different formation region.
Despite being widely separated, Fraser et al. (2017) showed that these blue binaries could survive being pushed outwards from their formation region by the migration of Neptune. The implication of this study is that the planetesimals that formed in the same region as these blue binaries must all have formed as binary systems. This is strong evidence for the formation of planetesimals and binaries through gravitational collapse, as only gravitational collapse is capable of producing a $\sim 100 \%$ binary fraction in addition to producing binaries with the properties discussed above.

By analysing high-resolution hydrodynamic simulations, Nesvorný et al. (2019) were able to study the properties of pebble clumps formed by streaming instability in detail. These clumps would then undergo gravitational collapse and can efficiently form binary systems. The authors showed that the orientations of these clumps matched the observed orientations of the TNBs, in particular, the 4:1 preference for prograde binary orbits as shown by Grundy et al. (2019). Only the combination of streaming instability and gravitational collapse can form binaries with this particular inclination distribution, thus making it the favoured mechanism to form TNBs and planetesimals.

The New Horizons mission has further stoked interest in the mechanisms of binary formation. After visiting the PlutoCharon system, New Horizons performed a flyby of the cold classical transneptunian object, $2014 \mathrm{MU}_{69}$ (Stern et al. 2019), now known as 486958 Arrokoth. This event was significant as this was the most distant spacecraft flyby to date and the first flyby of a Kuiper belt object. Being a cold classical object, Arrokoth is dynamically unexcited, and is believed to be composed of primitive material that has not been significantly altered since its formation. This makes it the perfect tool for probing accretion processes in the early Solar System. Imaging from the flyby revealed that this small object is composed of two distinct, similar-size lobes in a contact binary configuration. The implication is that the bi-lobate shape of Arrokoth must be primordial, and as discussed by Stern et al. (2019) and McKinnon et al. (2020), one of the favoured explanations is the formation of Arrokoth through gravitational collapse. This mechanism provides a path for producing bound planetesimals with nearly equal masses in a low-velocity collisional environment.

In this work we build upon the results of Nesvorný et al. (2010). We study the formation of binary systems through gravitational collapse in an independent manner, recreating the simulations within a different numerical framework using the REBOUND package. We perform our own detailed analysis, conducting a deeper search for bound particles, and take an in-depth look at the properties of the binary systems that are formed. The structure of the paper is as follows: firstly, we describe the setup for our gravitational-collapse simulations using the REBOUND package, the search for bound systems, and the dynamical evolution of any systems formed. We then present our results and perform a detailed analysis of the properties of the binary systems produced by our simulations. We compare our results to previous work and also to the latest data on the observed TNBs, and discuss the implications and limitations of our work, in particular, the effects of particle-size inflation and timestep-dependent collision detection.

\section{Methods}

\subsection{Simulation with REBOUND}

We set up our collapsing cloud simulations with the open source $N$-body package REBOUND (Rein \& Liu 2012). We used the 
symplectic epicyclic integrator within the rotating Hill frame, which is a fixed-timestep, second-order mixed variable symplectic method (Rein \& Tremaine 2011). An oct-tree algorithm (Barnes \& Hut 1986) was used to calculate gravity and to search nearest neighbours for collisions. We used the built-in openmp functionality to parallelise and take advantage of the strong scaling of the code. Collisions were resolved as simple inelastic mergers. We used open boundary conditions, where particles were removed if they left the simulation box. The simulation box was chosen to be sufficiently large such that only particles ejected from the cloud were removed: box size $=20$ cloud radii. The simulation state was saved at regular intervals. These timestamps allowed us to investigate the process of cloud collapse up until the maximum simulation time of $t=100 \mathrm{yr}$.

\subsection{Initial conditions}

For our initial conditions we adopted the values in Nesvorný et al. (2010). In summary, a spherical uniform cloud of $N_{\mathrm{p}}=10^{5}$ particles was defined by the total cloud mass $M_{\mathrm{c}}$, with cloud rotation modelled as the rotation of a solid body with angular frequency $\Omega$. Different rotation states were investigated, defined by the cloud rotation relative to the orbital angular frequency of a particle in a circular orbit at the edge of the cloud $X=\Omega / \Omega_{\text {circ }}$, where $\Omega_{\text {circ }}=\left(G M_{\mathrm{c}} / R_{\mathrm{c}}^{3}\right)^{1 / 2}$ and $R_{\mathrm{c}}$ is the cloud radius. Particle size was controlled by an inflation factor $f^{*}$, such that the modelled particle radius was $r_{f}=f^{*} r^{*}$, where $r^{*}$ is the radius of a uniform sphere of mass $m_{0}=M_{\mathrm{c}} / N_{\mathrm{p}}$ and density $\rho$. Throughout this work we have assumed a material density of $\rho=1000 \mathrm{~kg} \mathrm{~m}^{-3}$ to represent icy transneptunian material. The inflation factor, $f^{*}$, was included as a means of boosting the collision rate of the simulations and to ensure collisional damping of random velocities during collapse. Otherwise, given the limited number of computational particles and the therefore low particle number density, the growth of particles by accretion is significantly slower than the collapse timescale.

Nesvorný et al. (2010) explored 240 initial states: cloud masses spanning orders of magnitude $M_{\mathrm{c}}=4.19 \times 10^{18}, 6.54 \times$ $10^{19}$, and $1.77 \times 10^{21} \mathrm{~kg}^{1}$, with rotation states ranging from subto super-circular rotation $X=0.5,0.75,1.0$, and 1.25 , and a range of particle inflation factors $f^{*}=1,3,10,30$, and 100. Each combination of states above was repeated using four unique particleposition seeds. We dropped the $f^{*}=1$ and $X=1.25$ states, so that our dataset has 144 initial states. We ignored these cloud initial states as Nesvorný et al. (2010) discussed that these parameters are not conducive to forming binary objects. Our initial investigations of this region of parameter space showed similar trends. The self-gravity of clouds with $\Omega>\Omega_{\text {circ }}$ is not enough to overcome their rotation, therefore those clouds do not efficiently collapse and are dispersed due to excess angular momentum. Particles in clouds with $f^{*}=1$ have an extremely small collisional cross section, therefore particle growth by merging collisions is minimal over the 100-yr timescale we investigated.

The particle positions were generated by randomly and uniformly distributing $N_{\mathrm{p}}$ particles in the volume enclosed by a unit sphere. These seed positions were then rescaled according to the cloud mass at the start of each simulation, resulting in particle positions $\boldsymbol{r}=(x, y, z)$ distributed in a spherical volume of radius $R_{\mathrm{c}}=0.6 R_{\mathrm{Hill}, \mathrm{c}}$, where $R_{\mathrm{Hill}, \mathrm{c}}=\left(G M_{\mathrm{c}} / 3 \Omega_{\mathrm{Kep}}^{2}\right)^{1 / 3}$ is the Hill radius of the cloud at a heliocentric, Keplerian orbital frequency

1 These cloud masses are equivalent to a spherical body, uniform density $\rho=1000 \mathrm{~kg} \mathrm{~m}^{-3}$, of radius $R_{\mathrm{eq}}=100,250$, and $750 \mathrm{~km}$, respectively. of $\Omega_{\mathrm{Kep}}$, and the factor 0.6 ensures that all particles start bound within the cloud.

Initial particle velocities, $\boldsymbol{v}=\left(v_{x}, v_{y}, v_{z}\right)$, are the sum of the rotational velocity of the cloud and the Keplerian shear velocity due to the rotating Hill reference frame,

$\boldsymbol{v}=\boldsymbol{v}_{\text {rot }}+\boldsymbol{v}_{\text {shear }}$.

The angular velocity of the cloud is given by $\Omega=X \Omega_{\text {circ }}$, and the cloud rotates about the $\hat{z}$-axis, therefore $\boldsymbol{\Omega}=X \Omega_{\text {circ }} \hat{z}$. The rotational velocity component is then $\boldsymbol{v}_{\text {rot }}=\boldsymbol{\Omega} \times \boldsymbol{r}$. The Keplerian shear (Nakazawa \& Ida 1988) is described by

$\boldsymbol{v}_{\text {shear }}(x, y, z)=\left(0,-1.5 \Omega_{\text {Kep }} x, 0\right)$

for a rotating reference frame on a circular orbit with $\Omega_{\mathrm{Kep}}=\left(G M_{\odot} / a_{\mathrm{hel}}^{3}\right)^{1 / 2}$, where $a_{\mathrm{hel}}=30 \mathrm{AU}$ is the heliocentric semimajor axis of the cloud orbit. This distance is typical for the location of the primordial disc where the planetesimals formed (Morbidelli et al. 2008; Morbidelli \& Nesvorny 2020).

\subsection{Choosing the timestep}

We chose our simulation timestep to be small enough to accurately calculate particle trajectories, and to consistently detect collisions between particles. In REBOUND, a collision is recorded when two particles are found to be overlapping at the end of a timestep. The distance a particle moves in a single timestep must be smaller than the target particle size, otherwise an impacting particle could miss the target. We defined the relative collisional step size parameter as

$d_{\mathrm{rel}}=\mathrm{d} t \cdot v_{\mathrm{rel}} / d_{f, i}$,

where $\mathrm{d} t$ is the timestep, $d_{f, i}$ is the diameter of the target simulation particle $i$, and $v_{\text {rel }}$ is the relative (collisional) velocity between the target and impactor. We therefore defined a collision resolution criterion, $d_{\text {rel }}<1$, such that when a collision satisfies this inequality we assume that it has been well resolved. In Sect. 4.2.4 we consider the effects of increasing the timestep on mass accretion during gravitational collapse.

We chose the timestep for each simulation from an expected maximum collisional velocity and the initial particle size $d_{f}=2 r_{f}=2 f^{*} r^{*}$ such that

$\mathrm{d} t=\frac{1}{3} \frac{d_{f}}{v_{\max }}$

where we have included a numerical factor of $1 / 3$ to guarantee the criterion is met, and we have assumed that the maximum collisional velocity during collapse is $v_{\max }=30 \mathrm{~m} \mathrm{~s}^{-1}$. This estimate of the collisional velocity was obtained from Fraser et al. (2017), supplementary Fig. 4, but dynamical excitation of particles in the cloud during collapse is highly dependent on the initial cloud mass, with higher mass clouds having higher particle velocities.

To ensure that we achieved accurate collision resolution during gravitational collapse, we verified for each simulation that the relative collisional step size parameter (Eq. (3)) of all recorded collisions satisfied the collision resolution criterion. In most cases the criterion was well satisfied, thanks to our conservative choice of safety factor in Eq. (4). However, in some simulations of the highest cloud mass, $M_{\mathrm{c}}=1.77 \times 10^{21} \mathrm{~kg}$, we found that a moderate number of the collisions exceeded the estimated maximum velocity and so were detected outside the collision criterion. This meant that by chance we had detected 


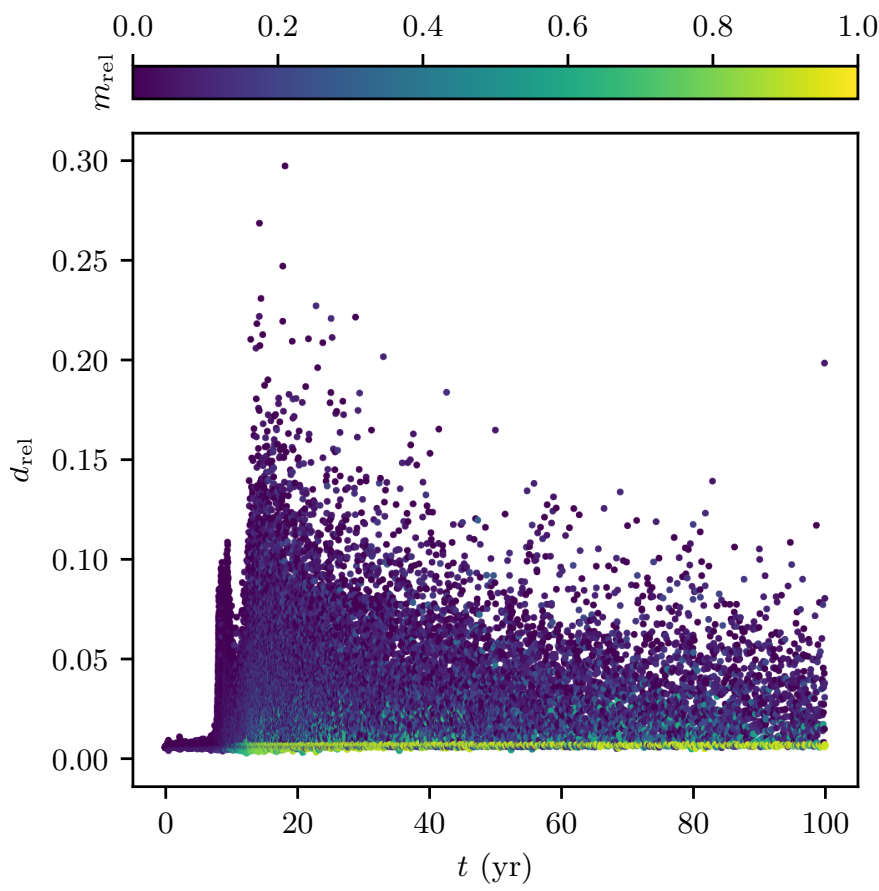

Fig. 1. Relative collisional step size (Eq. (3)) as a function of time for an example simulation $\left(f^{*}=10, X=0.5\right.$ and $\left.M_{\mathrm{c}}=6.54 \times 10^{19} \mathrm{~kg}\right)$. Each point represents a collision recorded in this collapsing-cloud simulation. The logarithmic colour scale, $m_{\text {rel }}=\log \left(m / m_{0}\right) / \log \left(M_{\mathrm{c}} / m_{0}\right)$, shows the mass of the primary (most massive) particle that is involved in each collision.

collisions that were faster than expected, which indicated that there could be other collisions that were missed. Therefore for these particular high cloud mass runs we decreased $\mathrm{d} t$, reducing the numerical factor in Eq. (4) from $1 / 3$ to $1 / 6$, and we repeated the simulations. When the collision criterion was tested for the final dataset, we found that only $0.002 \%$ of all recorded collisions had $d_{\text {rel }}>1$.

Even if a small number of collisions are missed, this does not invalidate our results. With gravitational collapse runaway growth is common, whereby large particles grow at a faster rate than smaller ones (Kokubo \& Ida 1996), quickly accreting the majority of the cloud mass. These are the particles that are most likely to harbour binary components, and in this study, only the 100 most massive simulation particles were searched for orbits (Sect. 3.1). The most massive particles were found to always be well within the collision criterion. Because the criterion depends on particle size, as $d_{f, i}$ increased, the criterion became easier to satisfy. This is demonstrated in Fig. 1, where we show the relative step size parameter for all collisions in an example gravitational-collapse simulation. We found that the collisions that fell outside the criterion were always between the smallest particles in the simulation, with $d_{f, i}$ close to the initial particle size. These fast collisions only occurred after the initial collapse of the cloud, given by the free-fall time

$t_{\mathrm{ff}}=\sqrt{\frac{3 \pi}{32 G \rho_{\mathrm{c}}}}$,

where $G$ is the gravitational constant, $\rho_{\mathrm{c}}=3 M_{\mathrm{c}} / 4 \pi R_{\mathrm{c}}^{3}$ is the initial cloud density, and $t_{\mathrm{ff}}=2.5 \times 10^{8} \mathrm{~s} \simeq 8 \mathrm{yr}$ for our initial conditions. By this stage the bulk of the cloud mass is already located in the most massive particles, therefore we argue that it is inconsequential that there may be a small number of undetected collisions between these small particles. The large bodies that form in the early phases of gravitational collapse are those that we are most interested in as binary system candidates. These bodies dominate the cloud mass fraction and have the greatest gravitational influence, therefore collisions between small bodies will not affect the already accreted larger bodies.

\subsection{Collision resolution}

We used the REBOUND collision tree module. This module uses an oct-tree to search for nearest neighbours and records a collision when overlapping particles are detected. Each simulation recorded a $\log$ of all collisions that contained the time and the target, impactor, and merged particle coordinates. We used the default inelastic merger collision routine, where the colliding particles are replaced by a single particle at the centre of mass of the original two. The resultant particle conserves total linear momentum, mass, and volume of the constituent particles.

In additional tests, Nesvorný et al. (2010) introduced inelastic bouncing collisions. Particles would merge if the impact velocity was lower than a certain threshold, otherwise, they would inelastically bounce with a loss of energy. This was found to have little effect on their simulations, as the majority of collisions were below the threshold velocity, therefore we also ignored such effects. To justify this assumption, we evaluated all recorded collisions against the merging collision criterion $v_{\text {rel }}<v_{\text {esc }}$ (Leinhardt \& Stewart 2012; Mustill et al. 2018), where $v_{\text {esc }}$ is the escape velocity of colliding particles with masses $m_{i}$ and $m_{j}$. We found that $87 \%$ of all recorded collisions satisfied this criterion and should indeed be merging. It should be noted, however, that in these simulations collisions are detected when the inflated particle radii overlap. We assessed the increase in collisional velocity if particles continue to accelerate towards each other and collide at their "true" physical radii $\left(r=(3 m / 4 \pi \rho)^{1 / 3}\right)$. The percentage of merging collisions drops to $79 \%$. For this estimate, we have assumed that all collisions are head-on. As all collisions have some non-zero impact parameter, our estimate represents an upper limit to the increase in collisional velocity. Regardless, the majority of collisions are still merging. The most massive particles (high $v_{\text {esc }}$ ) always experienced merging collisions, and it was only the low-mass particles at $t>t_{\mathrm{ff}}$ that could have bounced.

Furthermore, work by Sugiura et al. (2018) shows that collisions between planetesimals (albeit basalt ones) can be merging with no mass loss over an even wider range of impact velocities, depending on the impact angle. Therefore we assumed that inelastic merging collisions are appropriate when investigating gravitational collapse of the cloud masses used here. Bouncing collisions should not affect the formation of the most massive particles or any binary systems they may form.

\section{Results}

\subsection{Searching for bound systems}

After $t=100 \mathrm{yr}$, we halted the gravitational-collapse simulation and searched for bound systems of particles. We searched for orbits amongst a subset of simulation particles using the following criteria.

We are primarily interested in bodies that have undergone significant growth during the simulation; these are the objects that have condensed out of the particle cloud and formed distinct bodies. First we considered only particles that have had 
at least one merging collision, and had mass $m \geq 2 m_{0}$. The maximum mass of a binary system we could have detected is then $m_{1}+m_{2} \geq 4 m_{0}$. In general, at the end of gravitational collapse, most of the cloud mass was highly concentrated in only a handful of objects, and these particles are the most likely to have a companion. We also chose to ignore any bound systems in which the satellite would be extremely small and unobservable. To do this, we applied a mass ratio cut of $m / M>10^{-3}$ to the list of particles to be searched, where the most massive particle in the cloud had mass $M$. This is equivalent to an observational magnitude difference of $\Delta \mathrm{m}=5 \mathrm{mag}$ for a primary and secondary of the same density and albedo. Finally, we limited the orbit search to the $N_{\lim }=100$ most massive particles to ensure a fast and efficient search. This was the most significant constraint on the particles included in the orbit search but was appropriate, as explained in Sect. 4.2.3.

We took each of these particles to be a potential primary, and we searched all other particles in the subset to see if they were gravitationally bound. We first transformed the positions and velocities from the rotating Hill frame into the heliocentric frame. Then the orbit of the secondary relative to the primary was found using the REBOUND calculate_orbit function, assuming that this was a bound orbit if the binary eccentricity was $0 \leq e_{\text {bin }}<1$. This provides us with a list of bound orbits between pairs of particles. This list was sorted into independently bound systems of particles using the networkx package (Hagberg et al. 2008), which found all groups of mutually associated orbits. This method automatically accounts for all possible bound orbit architectures, for instance, several satellites orbiting a single primary or more complicated nested systems. Furthermore, it clearly shows when more than one bound system is produced in our simulations, that is, we detected no connecting orbits between independently bound systems.

At $t=100 \mathrm{yr}$, we found that it was common for the collapsed cloud to contain several bound systems, some of which showed a high degree of multiplicity. We detected a wide variety of bound systems, ranging from simple binary pairs to multiple-body systems of various flavours, for example, a single massive primary with a swarm of secondaries or circumbinary systems. We chose to include all systems of independently bound particles found by the orbit search in our analysis, unlike Nesvorný et al. (2010), who considered only the single most massive binary produced by each collapsing cloud. The orbits detected at this stage are instantaneous (osculating) orbital elements, and these systems may not be stable over longer timescales. It is therefore important to test these bound systems further.

\subsection{Further dynamical evolution of bound systems}

As in Nesvorný et al. (2010), we evaluated the stability of these systems by dynamically evolving them for a further $10^{4} \mathrm{yr}$. For each independently bound system of particles detected at $t=100 \mathrm{yr}$, a new $N$-body simulation was launched to investigate its dynamical stability. We integrated the system in the heliocentric frame using the REBOUND leapfrog integrator. During the previous gravitational-collapse simulations the most massive particles grew quickly by runaway growth, and so by $t=100 \mathrm{yr}$, their mass accretion was complete. We therefore removed the inflation factor $f^{*}$ for these integrations; particles of mass $m$ were initialised with radius $r=(3 m / 4 \pi \rho)^{1 / 3}$. Size inflation was important for boosting the collision rate during gravitational collapse but it prevents us from investigating the dynamics of tight orbits, as particles cannot get close without colliding and merging into a single body.
After the additional integration the orbit search was repeated, and we assessed how the initially bound system had evolved. In this dataset we included only orbits that had $a_{\text {bin }} / R_{\text {Hill }}<0.5$, where $a_{\text {bin }}$ is the binary orbit semimajor axis, and the Hill radius of the binary primary of mass $m_{1}$ is

$R_{\text {Hill }}=a_{\text {hel }}\left(m_{1} / 3 M_{\odot}\right)^{1 / 3}$,

where $a_{\mathrm{hel}} \simeq 30 \mathrm{AU}$ is the heliocentric distance of the binary primary. This was to filter out any poorly bound orbits that we did not expect to survive.

At $t=100 \mathrm{yr}, 287$ bound systems were detected for the 144 collapse simulations. Of these systems, $55 \%$ were simple binary pairs and the remainder were multiple-body systems. After $10^{4} \mathrm{yr}$ of dynamical evolution we found that either multiplicity $N$ was greatly reduced (most systems evolved to simple binary pairs), or the system was destroyed (either dynamically or collisionally). We were left with 223 systems: 188 binary systems, and 35 with $N>2$. Of the systems that were initially binary pairs, $67 \%$ had survived, and $63 \%$ of the multiple systems had evolved to become $N=2$ binaries. For the surviving multiple-body systems, 32 had been reduced to triple systems, and 3 systems had $N=4$ bound particles.

\subsection{Properties of binary systems}

For our analysis of the bound systems produced by gravitational collapse we focused on the binary planetesimals. After the dynamical evolution, some multiple particle systems $(N>2)$ survived in our dataset, but with even further dynamical evolution these systems may be destroyed, evolve into binaries, or may remain as stable $N>2$ systems. In the following analysis, we only briefly assess the properties of the multiple-body systems by considering the orbit between the two most massive particles in each system. We made the assumption that the properties of the most massive particles would not be greatly changed by future evolution, provided the system survives.

\subsubsection{Binary masses}

In Fig. 2 we plot the binary mass ratio (secondary mass/primary mass) against the total binary mass normalised by initial cloud mass in log-log space. A wide range of systems are formed, but there are three distinct populations, which we classify as follows:

1. Particles that have undergone minimal mass accretion: $\left(m_{2}+\right.$ $\left.m_{1}\right) / M_{\text {cloud }} \sim 10^{-3}$, but can have high mass ratios. We refer to these as "atomistic binaries".

2. Particles with negligible mass companions: $m_{2} / m_{1} \sim 10^{-2.5}$. We call these the "satellite systems".

3. Particles that have undergone moderate to high mass accretion and have reasonably sized companions, $\left(m_{2}+\right.$ $\left.m_{1}\right) / M_{\text {cloud }} \gtrsim 0.1$ and $m_{2} / m_{1} \gtrsim 0.1$. We classify these as "observable binary" systems.

The orbit search (Sect. 3.1) detected a wide range of mass ratios, from $\sim 10^{-3}$ (which is the cut-off for our orbit search) up to exactly 1 . The binaries with $m_{2} / m_{1}=1$ are composed of particles that have had the exact same number of merging collisions. Approximately $50 \%$ of the binary systems have $m_{2} / m_{1}>0.1$, which corresponds to a size ratio of $r_{2} / r_{1}=\left(m_{2} / m_{1}\right)^{1 / 3}=0.46$. Gravitational collapse does not necessarily have a preference to form similar size binaries. The binary system masses span a range from $6 \times 10^{-5} M_{\mathrm{c}}$ up to $0.75 M_{\mathrm{c}}$.

To test for the presence of these classes, we performed a Scikit-learn DBSCAN cluster search (Ester et al. 1996; 


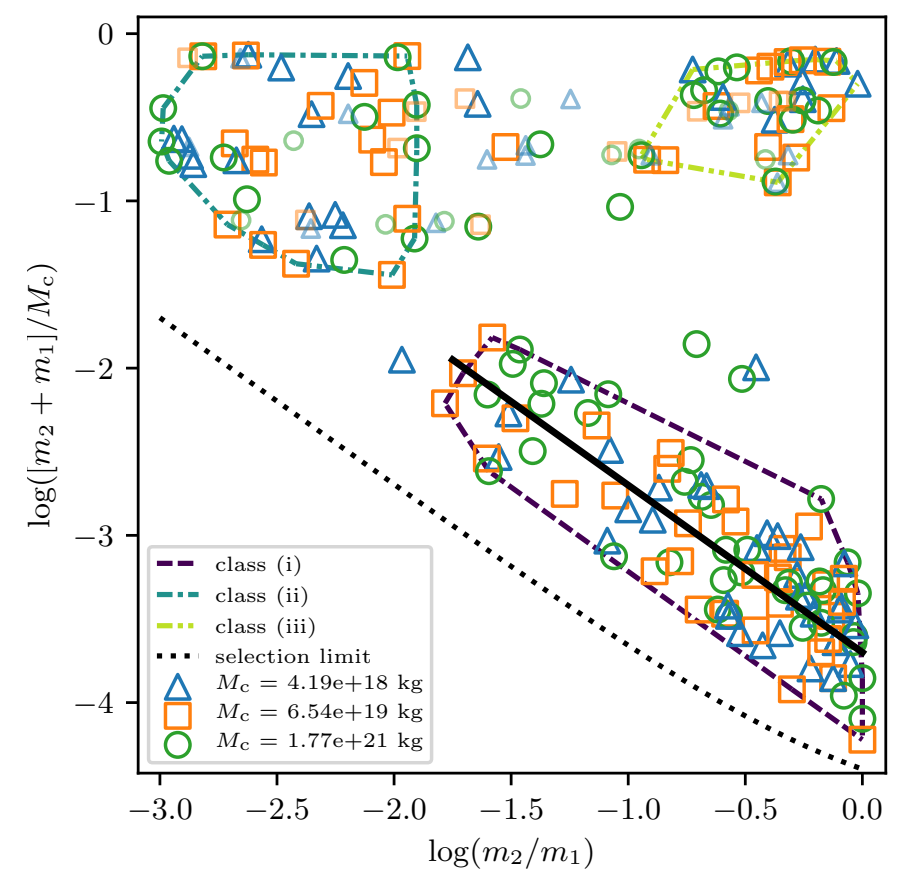

Fig. 2. Binary system mass normalised by initial cloud mass $\left(m_{2}+\right.$ $\left.m_{1}\right) / M_{\mathrm{c}}$, against the secondary-to-primary mass ratio, $m_{2} / m_{1}$, plotted in $\log -\log$ space. We display the results for all values of $M_{\mathrm{c}}, X$, and $f^{*}$ in our dataset. Marker colour and shape denote the three initial cloud masses. Dashed coloured lines trace the boundary points of the three binary classes. The solid line was drawn to guide the eye to the linear trend of the class (i) particles. The detection limit for our orbit search algorithm is shown as the dotted line. We also include data points representative of the $N>2$ bound systems. The total mass and mass ratio for the two largest particles in these systems are plotted, represented by the smaller, fainter markers than the binaries.

Pedregosa et al. 2011) on the binary dataset features, $\log \left(\left[m_{2}+\right.\right.$ $\left.\left.m_{1}\right] / M_{\mathrm{c}}\right)$ and $\log \left(m_{2} / m_{1}\right)$. These features were first standardised, that is, the mean was removed and the data scaled to unit variance. DBSCAN then finds clusters by grouping particles that have a minimum number of neighbours within a distance parameter; in this search we used the default values 5 and 0.5 for these parameters, respectively. The algorithm found the three proposed classes with a small number of outliers. It is clear that the distribution of the data is invariant with different cloud masses and that the class (i) atomistic binaries follow a linear trend; a line is drawn on Fig. 2 to guide the eye. It may be possible that this trend extends to lower mass ratios for a subset of the satellite systems, but for now we consider only the atomistic binaries. This trend suggests that these objects follow a power-law relation between the binary system mass (i.e. the mass accretion efficiency of gravitational collapse) and the binary mass ratio of the form

$\frac{m_{2}}{m_{1}}=10^{-b / s} \alpha^{1 / s}$

where we define the mass accretion efficiency as $\alpha=\left(m_{2}+\right.$ $\left.m_{1}\right) / M_{\mathrm{c}}$. This linear trend (in log-log space) can be approximated by a gradient and intercept of $s=-1.0$ and $b=-3.7$ respectively, such that $m_{2} / m_{1} \propto \alpha^{-1}$.

This either indicates an underlying physical relation between mass accretion and mass ratio for the atomistic binaries formed during gravitational collapse, or that there are unknown biases in our simulation method. To investigate this, we considered the selection limit of the orbit search algorithm. As described in
Sect. 3.1, we required that both components had undergone at least one merging collision. Particles must have $m \geq 2 \times 10^{-5} M_{\mathrm{c}}$ to be searched, therefore the lowest detectable binary system mass is $4 \times 10^{-5} M_{\mathrm{c}}$. As we also imposed $m_{2} / m_{1} \geq 10^{-3}$, there is a limit to the lowest secondary mass that our algorithm will detect for a given primary mass, which we show as a dotted line in Fig. 2. However, all binaries in our dataset are above this selection limit. It is also possible that this trend could be a numerical artefact of the $N$-body method used in this study. Higher resolution simulations with more particles are required in order to verify this trend. On the other hand, if this relationship is truly physical, it implies that gravitational collapse produces some binaries where the mass ratio depends primarily on the collapse accretion efficiency. This is described by Eq. (7), with exact coefficients that depend on the (currently approximated) slope of the trend line. Thus this trend may provide an observationally detectable signature indicating cloud collapse as a real formation route for binary systems.

We then evaluated the $N>2$ systems, which make up $16 \%$ of our dataset. In Fig. 2 we include data points that represent what a multiple-body system would look like as a binary if we considered only the properties of the two most massive particles in the system. We made this assumption as most multiples in our dataset were in a circumbinary configuration, where a tight inner binary is orbited by a more distant lower mass satellite. Unlike the binary pairs, which generally had high mass ratios, the $N>2$ systems all had relatively low mass ratios. The median mass ratio for the two most massive particles in these systems was $m_{2} / m_{1}=0.03$, therefore we would expect only minor gravitational perturbations from the third (or fourth) body. It is likely that such a system could initially be discovered as a binary, as the inner most satellite may be unresolved, or that a faint, distant satellite is below the detection limit. With further observation, it could be revealed that what appears to be a binary is actually a multiple system, for example $1999 \mathrm{TC}_{36}$ Lempo (Benecchi et al. 2010). In Fig. 2 the $N>2$ systems fall into the mass range of observable binaries and satellite systems, that is, the main orbit of a multiple-body system contains a reasonable fraction of the collapsing cloud mass. The mass ratios between the two largest bodies, however, span the full range of possible values. Multiple-body systems appear to bridge the gap between the two classes, adding to the population of outlier points. We repeated the DBSCAN cluster search for both binaries and multiples using the same parameters as before, and found that classes (ii) and (iii) were detected as a single large cluster. Hence it is possible that observationally, only these two groupings would be detected, although we note that in Fig. 2 the high-multiplicity systems more frequently have either high or low $m_{2} / m_{1}$. These systems would likely fall into the observable binary and satellite system categories. Moreover, we did not consider how an $N>2$ system might evolve for timescales longer than $10^{4} \mathrm{yr}$. Further integration is required to investigate the stability of such systems over longer timescales to the present day. Collisions between components may change the mass ratios, a component may be ejected, or the system could be destroyed altogether.

The data (binary orbits only) separated by the different values of particle inflation factor $f^{*}$ are shown in Fig. 3. Marker size indicates the separation of binary components relative to the Hill radius of the primary (Eq. (6)). We see that simulations with $f^{*}=30$ produced the most observable binary systems. For low values of $f^{*}$, the particle collisional cross section is small and the reduced number of high-mass systems is explained by the low collision rate between particles. This is supported by the systems that are detected for $f^{*}=3$ and 10 mostly belonging to 


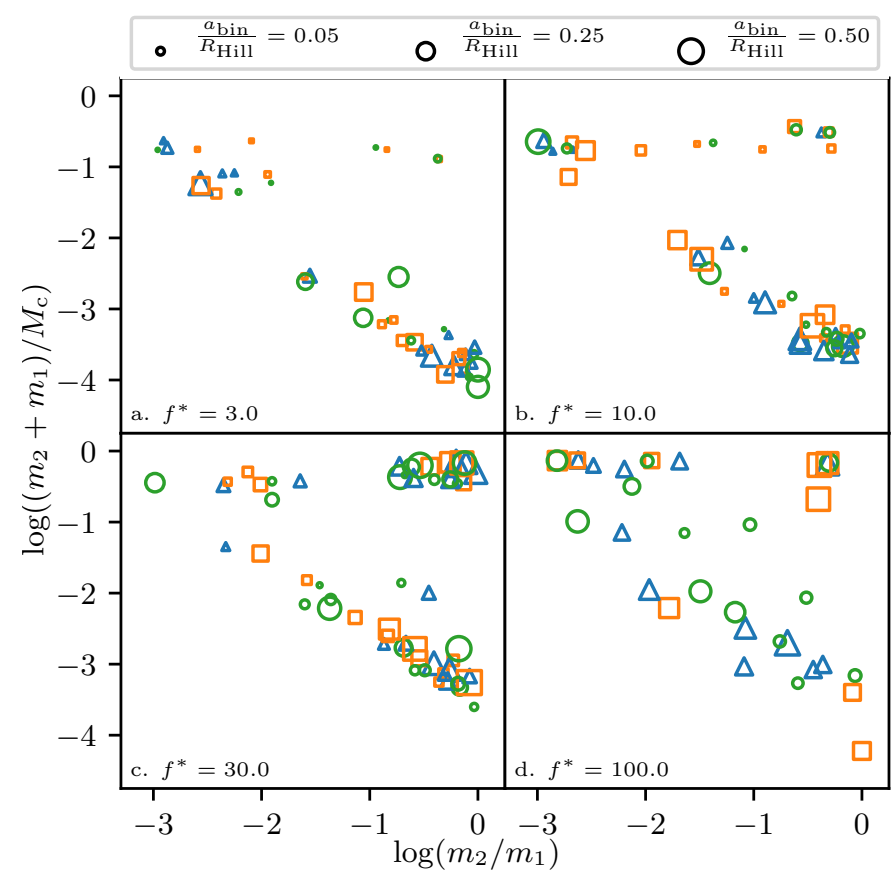

Fig. 3. Breakdown of Fig. 2 (binaries only) by particle inflation factor $f^{*}$. Marker colours and shapes are the same as before, and marker size scales with relative binary separation, $a_{\mathrm{bin}} / R_{\text {Hill }}$. Panels $a-d$ : binaries produced by simulations with $f^{*}=3,10,30$, and 100 , respectively.

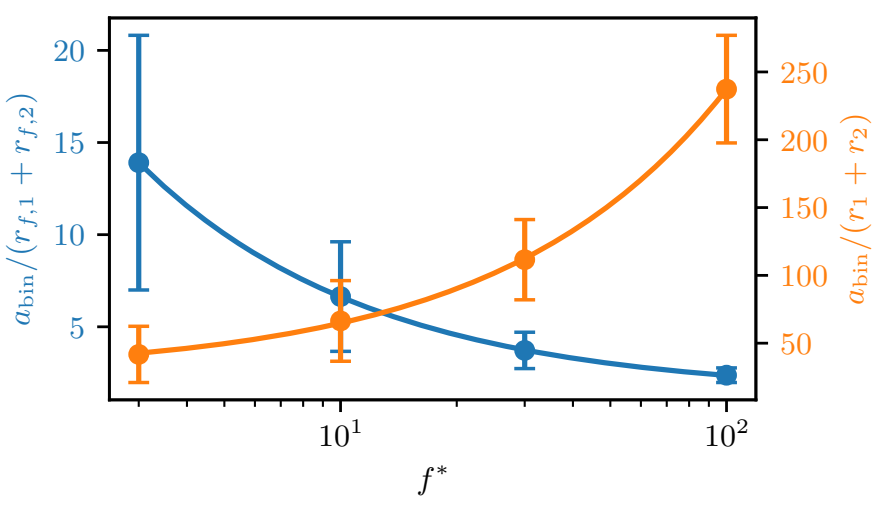

Fig. 4. Median binary separation relative to the particle size as a function of particle inflation factor $f^{*}$ used in the simulation that formed the binary. The left-hand axis (blue) shows separation relative to the inflated particle radii, and the right-hand axis (orange) is relative to the uninflated physical particle radii. The error bars indicate the standard error for each set of data points from simulations with a given $f^{*}$. Power-law fits are drawn to guide the eye.

the low-mass atomistic binary group. As $f^{*} \rightarrow 30$, collision rate and mass accretion increases and more high-mass systems with $m_{1}+m_{2}>0.1 M_{\mathrm{c}}$ are produced.

When $f^{*}=100$, the number of high-mass binaries drops. With high $f^{*}$, we would expect an increased collision rate, but instead we hit the limit where these extremely large inflated particles cannot form a tight binary without colliding and destroying the system. To show this, we considered the binary separation relative to the particle size, both with and without particle size inflation. In Fig. 4 we plot the median relative separation of all binaries formed by simulations with a particular value of $f^{*}$. When the increase in particle size due to $f^{*}$ is accounted for, the relative separation is given by $a_{\mathrm{bin}} /\left(r_{f, 1}+r_{f, 2}\right)$. Simulations

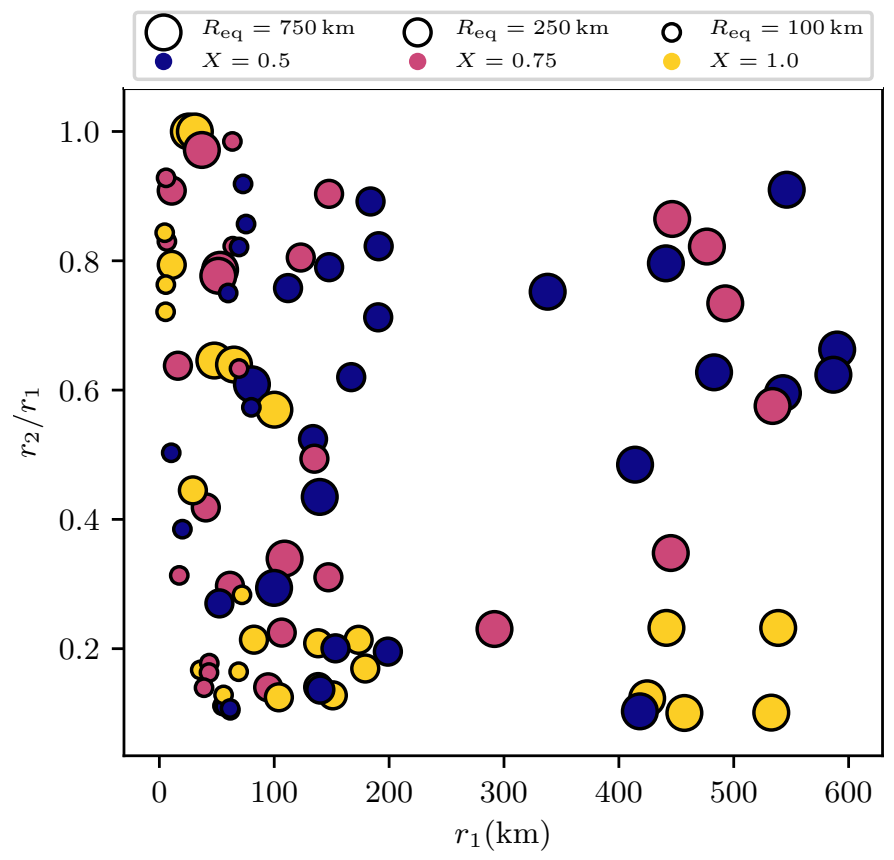

Fig. 5. Binary size ratio against primary size from our simulations, shown in a similar manner to Fig. 2 of Nesvorný et al. (2010). Marker size indicates the cloud mass (given as an equivalent size $R_{\mathrm{eq}}$ ), and colour gives the cloud rotation. As in the original, we show only the results for $f^{*}=3,10,30$.

with larger $f^{*}$ generally produce binaries that are tight relative to the inflated particle size (Fig. 4, blue line). This makes such systems more vulnerable to destruction by mutual collision. When the relative separation is recalculated, but instead using the physical particle radii, $a_{\text {bin }} /\left(r_{1}+r_{2}\right)$, the trend is now that relative separation increases for simulations run with higher values of $f^{*}$ (Fig. 4, orange line). This means that in addition to reducing the number of binaries formed (due to mutual collisions between components), simulations with higher values of $f^{*}$ are biased against producing binaries with tight orbits.

\subsubsection{Binary sizes}

To compare our data with the binary systems of Nesvorný et al. (2010) we recreated their Fig. 2, a plot of the binary secondary/primary size $r_{2} / r_{1}=\left(m_{2} / m_{1}\right)^{1 / 3}$ against the (uninflated) primary radius $r_{1}$ (Fig. 5). For consistency with their work, we only considered binaries produced by clouds with moderate values of inflation factor, $f^{*}=3,10,30$. However, we found that including the $f^{*}=100$ binary systems did little to change the overall distribution.

Comparing the figures, we note that our implementation of gravitational collapse produced more massive binaries. Nesvorný et al. (2010) produced a maximum primary radius of $r_{1} \simeq 400 \mathrm{~km}$ for the highest cloud mass, which corresponds to a fraction $(400 / 750)^{3}=0.15$ of the initial cloud mass. In contrast, the largest particle in Fig. 5 is approximately $(600 / 750)^{3}=0.5$ of the initial cloud mass. As shown in Fig. 2, we found that gravitational collapse generally accretes a large fraction (up to 75\%) of the cloud mass into a binary system, and that this process is invariant with $M_{\mathrm{c}}$. The cloud masses span three orders of magnitude, and if the binaries all accreted approximately the same mass fraction, the particle sizes will also cover a wide range. This explains why we have a less uniform distribution in $r_{1}$ than 
Nesvorný et al. (2010). This increased mass accretion efficiency in our implementation of gravitational collapse may be a result of enhanced collision resolution. As described in Sect. 2.4, we tailored the timestep for each cloud to ensure collisions were accurately detected. Nesvorný et al. (2010) used a longer, fixed timestep of 0.3 days for all cloud masses, but with a collision detection method that extrapolates particle trajectories to allow for longer timesteps (Richardson et al. 2000). We investigate this further in Sect. 4.2.4.

Furthermore, we found a wider spread in binary size ratio, but this is to be expected as we have included all binaries found by the deep orbit search. In Fig. 5 we reproduce the trend of Nesvorný et al. (2010) that most large, equal-sized binaries were produced by clouds with rotation $X<1$. In contrast, we found that some $X=1$ clouds were able to produce large systems, but these are all low size ratio satellite systems. At the other extreme, we detected binaries from $X=1$ clouds with small primaries and an equal-size secondary: these are the atomistic binaries. Although the initial angular momentum of the cloud would be most efficiently "spent" by forming a large similar-size binary, in the $X=1$ case we reach the limit where cloud collapse is resisted by the cloud rotation. Particles are more readily dispersed and ejected by the Keplerian shear, leading to a loss of angular momentum. Therefore satellite systems and atomistic binaries are preferentially formed in $X=1$ simulations. When we take into account the larger particles and wider variety of systems that we included in this study, our implementation of gravitational collapse produced a subset of systems of appreciable mass and equal-sized components, similar to Nesvorný et al. (2010).

\subsubsection{Binary magnitudes}

We then compared our binary systems with all the observed TNBs from the Grundy (2019) database (accessed 2 December 2019, 102 objects). Within this dataset some systems are classified as "special cases", for example the known triple system Lempo (Benecchi et al. 2010), the contact or nearly contact binary 2001 QG $_{298}$ (Sheppard \& Jewitt 2004; Lacerda 2011), and some are placeholders for known binaries with incomplete orbits. We excluded the unusual triple and contact systems. The dynamically complex Pluto and Haumea systems were not included in this dataset, but we did include dwarf planet systems such as Eris and Makemake, plus the "nearly certain" dwarf planet candidates: $2007 \mathrm{OR}_{10}$, Quaoar, Orcus, and Salacia (Brown 2019, accessed 3 December 2019).

Similar to Fig. 3 of Nesvorný et al. (2010), we evaluated the magnitude of the binary primary as a function of the primarysecondary magnitude difference for the simulated binaries and observed TNBs (Fig. 6). We used the uninflated radii ${ }^{2}$ for the primary and secondary particles, $r_{1}$ and $r_{2}$, to calculate an observed $V$-band magnitude for each binary system (Appendix A). Magnitudes were calculated at a heliocentric distance of $R=44 \mathrm{AU}$ to compare with the observed TNBs, most of which are located in the classical belt. We highlight that the gravitational-collapse simulations were set up at $30 \mathrm{AU}$, therefore we have to assume that either the binaries have migrated from their formation region, or that streaming instability and gravitational collapse are not strongly dependent on heliocentric distance (Yang et al. 2017). A geometric albedo of $p_{V}=0.15$ was used for both components, which is the observed median albedo for cold classical

2 Similar to Sects. 2.4 and 3.2, the uninflated particle radius was calculated from mass $m$ and density $\rho$. objects (Lacerda et al. 2014), whereas Nesvorný et al. (2010) used $p_{V}=0.08$. We also included data points representing the $N>2$ particle systems formed by gravitational collapse, as in Fig. 2 above. These data points were added to show what a multiple system may look like if it were to be initially discovered as a binary. Furthermore, in Fig. 6 we included a line representing an approximate empirical detection limit (see Noll et al. 2008, Fig. 3), and a line at magnitude $m_{V}=25$ to represent a rough observational brightness limit.

We also added a data point that is representative of the contact binary Arrokoth, with magnitudes calculated using the same method as the binaries above. The volumes of the primary and secondary components are $V_{1}=1400 \pm 600 \mathrm{~km}^{3}$ and $V_{2}=1050 \pm 400 \mathrm{~km}^{3}$, respectively (Stern et al. 2019). To compare with the simulated binaries, we assumed that the components are spherical and therefore have radii $r_{1}=6.9 \pm 1.0 \mathrm{~km}, r_{2}=6.3 \pm$ $0.8 \mathrm{~km}$. We considered the hypothetical case where these components are separately resolved such that we can calculate a primary magnitude and a magnitude difference. We assumed the same observational parameters for distance and albedo as used above. Compared to the true distance $R=44.6 \mathrm{AU}$ and albedo $p_{V}=0.165$ (Stern et al. 2019), the difference in magnitude is minimal.

Figure 6 shows that each cloud mass produced a similar distribution of binary systems, composed of a linear "fan" and a "clump". For each cloud mass these different size regimes are a result of the additional systems found by the deep orbit search (Sect. 3.1). The clump is composed of the observable binaries, which have high system mass and mass ratios. The linear fan is composed of atomistic binaries and satellite systems. We compare this to Fig. 2, where these classes were defined and the linear trend of the atomistic binaries was first noticed. It is interesting that the observed TNBs follow a similar linear trend as the fan, but we would expect that in this case it is due to detection limits and observational biases. In particular, high cloud mass binaries in the fan lie in a similar parameter space as the observed TNBs with large $\Delta \mathrm{m}_{V}$ (Fig. 6). We emphasise again that our binary data points are above the orbit-search selection limit (Fig. 2), and this implies that there is either a physical mechanism or simulation bias that causes this trend. Figure 2 also demonstrated that the distribution of binary mass properties is invariant when normalised by cloud mass. Figure 6 shows that each cloud mass follows a similar distribution, but with an offset in brightness. More massive clouds produce more massive, brighter binaries.

In Fig. 6 we find that the observable binaries from the low cloud mass and the atomistic binaries from the high cloud mass are the best match to the main cluster of observed TNBs with $\mathrm{m}_{V} \sim 22-25$ and $\Delta \mathrm{m}_{V} \sim 0-1 \mathrm{mag}$. In terms of having high system mass and high mass ratios, our observable binaries are comparable to the binaries detected by Nesvorný et al. (2010), who selected the single most massive binary from each cloud. For the atomistic binary systems, although they are a small fraction of the initial cloud mass, $M_{\mathrm{c}}=1.77 \times 10^{21} \mathrm{~kg}$ is orders of magnitude more massive than the other clouds. Therefore these two binary classes from different clouds occupy a similar size and brightness range.

In contrast, Nesvorný et al. (2010) found that the intermediate cloud mass best replicated the observed TNBs. This discrepancy arises firstly because the binaries produced in our gravitationalcollapse simulations are generally larger (Fig. 5), and we have used a higher albedo. This means that our binaries are brighter, which shifts the distribution upwards in Fig. 6, pushing our intermediate cloud mass observable binaries away from the region of 


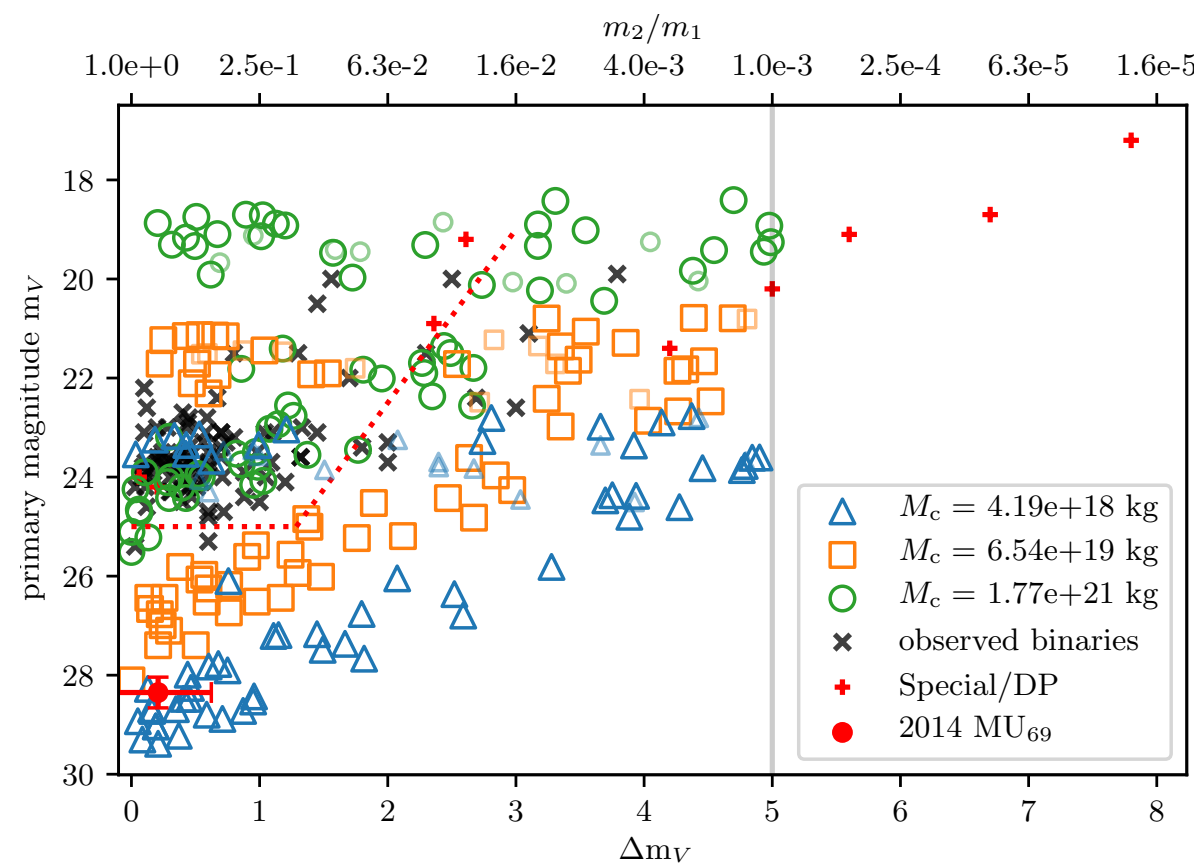

Fig. 6. Primary $V$-band magnitude against magnitude difference between primary and secondary for binaries produced by gravitationalcollapse simulations. Particle masses were converted into a spherical radius (assuming uniform density), which was then converted into a magnitude using a fixed albedo and distance. As with Fig. 2, marker colour and shape indicate the initial cloud mass, and any $N>2$ systems are represented by smaller, fainter markers. The mass ratio for a given magnitude difference is shown on the upper $x$-axis. The vertical line indicates the mass ratio cut-off of $m_{2} / m_{1} \geq 10^{-3}$ in the orbit-search algorithm. The observed TNBs from Grundy (2019) are shown as black crosses. We represent "special cases" and dwarf planets as red plusses. The red circle with error bars represents how Arrokoth (2014 $\mathrm{MU}_{69}$ ) would appear if its components could be separately resolved. An approximate empirical detection limit (Noll et al. 2008) and a lower magnitude limit of 25 are shown as dotted red lines. the observed TNBs. Secondly, we have investigated all bound systems produced by gravitational collapse, whereas Nesvorný et al. (2010) did not consider atomistic binaries.

We emphasise that there are many tunable parameters in this analysis, such as heliocentric distance, albedo, and density. For example, if the material density was decreased to $250 \mathrm{~kg} \mathrm{~m}^{-3}$ objects would appear $\sim 1$ mag brighter. On the other hand, we did not take possible increases in density caused by compaction from collisions or differentiation due to self-gravity into account. Furthermore, we have shown that the distribution of relative system mass and mass ratio is invariant with $M_{\mathrm{c}}$, therefore we expect that increasing or decreasing the initial cloud mass would shift the magnitude distribution to brighter or fainter values. Depending on the mass distribution of pebble clouds formed by streaming instability (Li et al. 2019; Nesvorný et al. 2019), clouds might be found whose binary masses match the observed magnitudes better.

Interestingly, Fig. 6 shows a distinct clump of simulated high cloud mass binaries that have no observational counterpart amongst the TNBs. These are objects that would have magnitudes comparable to the dwarf planets, with a similar size companion, and should be easily detected. If these objects are not observed, perhaps these binaries are preferentially destroyed by some mechanism. For example, tidal evolution may shrink the orbit until the components collide and merge into a single object. An additional factor is that it is relatively rare for the green points in Fig. 6 to lie in the clump, compared to the large number of binaries in the fan (12 out of 66 binaries are in the clump for the high-mass cloud dataset). A more likely explanation, however, is that such high cloud masses simply never existed, and therefore these objects could not be produced by gravitational collapse. First of all, most cold classicals are observed to have a characteristic diameter $d \simeq 140 \mathrm{~km}$, as this marks the break from the steep size distribution of larger objects (Fraser et al. 2014). Therefore high-mass clouds produce objects that are too large to match observations. Secondly, the streaming instability simulations of Nesvorný et al. (2019) produce a maximum cloud mass of $\sim 10^{21} \mathrm{~kg}$ (depending on disc parameters, Appendix B). From these estimates, we see that the highest cloud mass is probably too high to be easily formed by streaming instability. Therefore it seems unlikely that the high system mass, high-mass ratio clump of $M_{\mathrm{c}}=1.77 \times 10^{21} \mathrm{~kg}$ binaries shown in Fig. 6 can be formed.

We note that there is also the (rather disappointing) possibility that the observable binary feature may only arise due to our limited simulation method, in particular, the assumptions of inelastic merging collisions (Sect. 2.4) and particle size inflation (Sect. 4.2). We have justified these assumptions above, but ultimately, more accurate models of gravitational collapse (e.g. higher numerical resolution) are required to definitively confirm that the outcomes of these assumptions are indeed accurate reflections of reality.

\subsubsection{Ratio of binary to single planetesimals}

We also considered the number of particles produced by gravitational collapse, which may be singles or in a bound system, that would be above the observational limit of $\mathrm{m}_{V}=25$. In Fig. 6 this brightness is equivalent to a simulation particle mass of $m_{25}=1.4 \times 10^{17} \mathrm{~kg}$. The distribution of particle masses produced by gravitational collapse is a steep power-law, with a particle number that drops sharply with increasing mass. For each cloud in our dataset, we plotted the mass distribution of all particles in the simulation box at $t=100 \mathrm{yr}$, as shown in Fig. 7. By assessing $m$ in units of the initial particle mass, $m_{0}$, we see that all three cloud masses follow similar distributions. At low particle mass $\log \left(m / m_{0}\right)<2$, the mass distribution generally follows a power law of the form $\mathrm{d} n \propto m^{q} \mathrm{~d} m$, where $q=-2.1$, with roughly two orders of magnitude scatter about that power law. At higher masses the distribution flattens off; each simulation produces only a small number of particles that contain a high fraction of the initial cloud mass.

For the low, intermediate, and high cloud mass simulations, the mean number of single particles with a brightness of $25 \mathrm{mag}$ or greater is $n_{\text {single }}\left(m \geq m_{25}\right)=0.3,1.4$, and 147.2, respectively. When we consider our entire dataset (binaries and multiples), the 144 gravitational-collapse simulations produced 160 bound systems with $m_{\text {sys }} \geq m_{25}$. The low-, intermediate-, and high-mass clouds produced on average $n_{\text {bound }}\left(m_{\text {sys }} \geq m_{25}\right)=0.8$, 


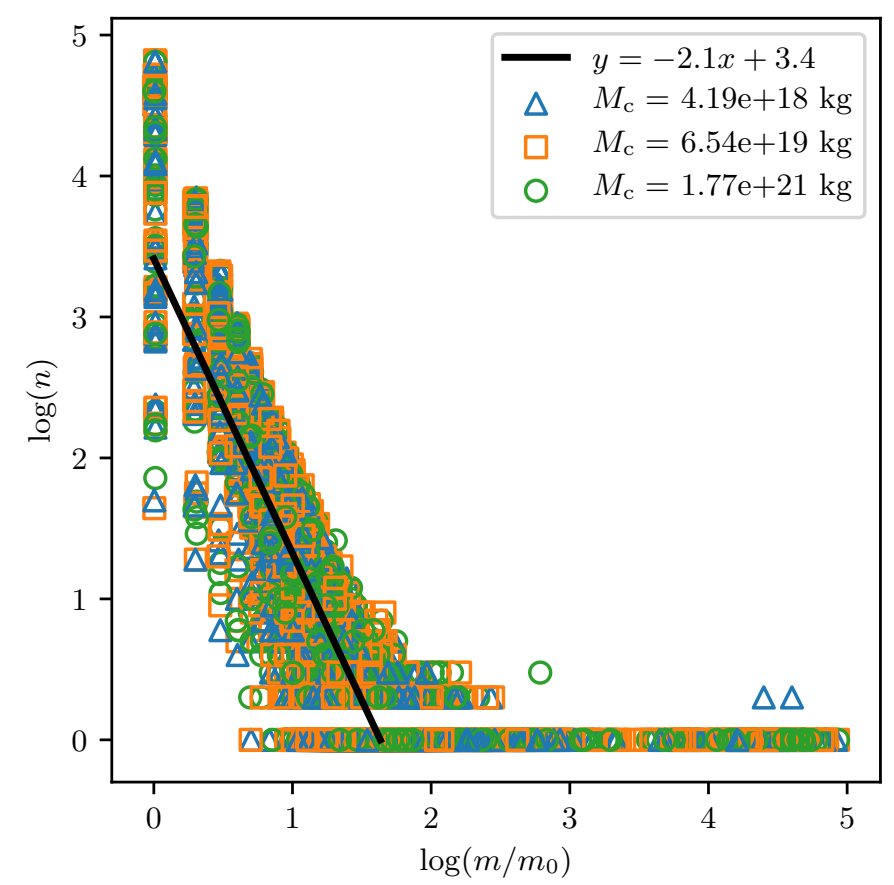

Fig. 7. Mass distribution of all particles in the simulation box at $t=100 \mathrm{yr}$. The number of particles $n$ of mass $m$, in units of the initial particle mass $m_{0}=M_{\mathrm{c}} / N_{\mathrm{p}}$, are shown in log-log space. Marker colour and shape denote the three cloud masses. A linear regression on all data points with $\log \left(m / m_{0}\right)<2$ is shown to guide the eye to the power-law distribution.

1.0, and 1.5 observable bound particle systems per cloud. Therefore, when we compare $n_{\text {single }}$ to $n_{\text {bound }}$, only the low cloud masses are compatible with forming nearly all observable planetesimals as binaries, as proposed by Fraser et al. (2017), with $\sim 2.6$ binaries produced for every single planetesimal. This is further evidence against the gravitational collapse of high-mass clouds forming TNBs, and supports an upper mass limit on clumps formed by streaming instability.

\subsubsection{Binary orbital elements}

The distribution of binary orbital elements produced by gravitational collapse: semimajor axis $a_{\text {bin }}$, eccentricity $e_{\text {bin }}$, and inclination $i_{\text {bin }}$ are shown in Fig. 8. These are compared to the 35 observed TNBs for which there are full orbital solutions (Grundy et al. 2019). For the observed TNBs the reported inclination is the angle between the mutual binary orbit and its heliocentric orbit. The simulated binary inclinations are measured relative to the $x y$ plane of the rotating reference frame, that is, relative to the heliocentric orbital plane of the pebble cloud. We see that each cloud produces a wide spread of binary orbits, spanning the full range of eccentricity and inclination. There is a trend for larger binary semimajor axis with increasing cloud mass, which is primarily caused by the longer mean free path of particles in more massive clouds. We have highlighted the orbital properties of the observable binaries with bold markers. These objects are analogous to the binary systems found by Nesvorný et al. (2010); they generally have low prograde inclinations and low to moderate eccentricities.

We then reproduced Fig. 1 of Grundy et al. (2019), a polar plot of binary mutual inclination on the azimuthal axis against the relative separation on the radial axis for the 35 observed TNBs with full orbit solutions (Fig. 9). For comparison we

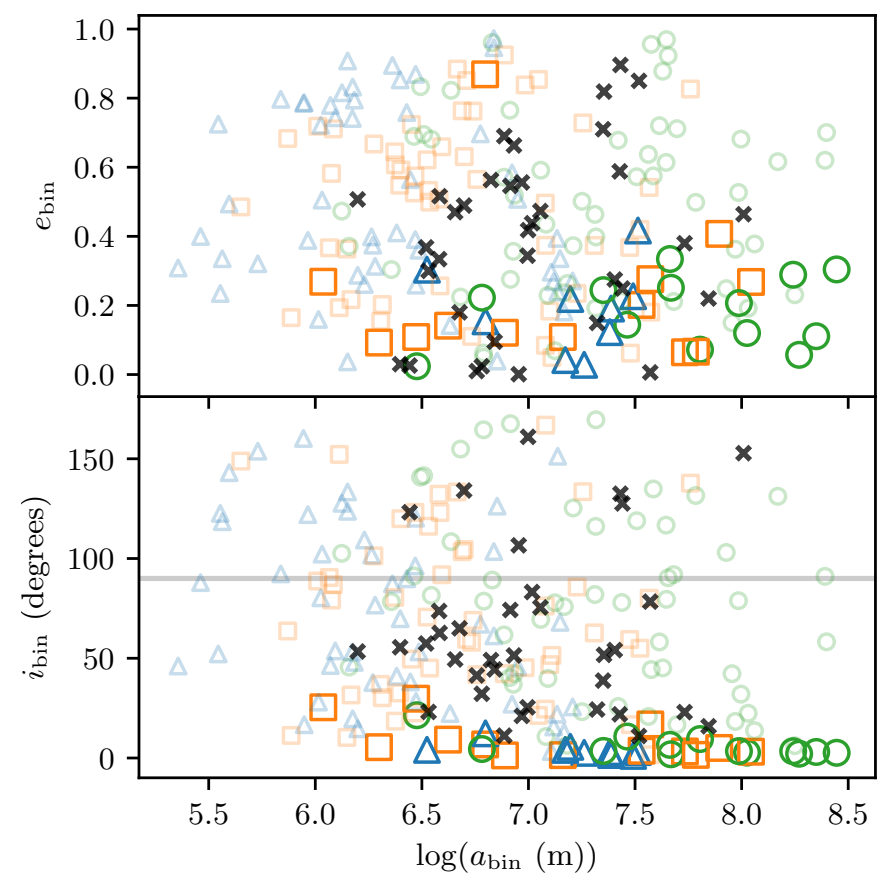

Fig. 8. Binary orbital parameters for the simulated binaries compared to the 35 observed TNBs (black crosses) with full orbit solutions (Grundy et al. 2019, Table 19). Again, marker shape and colour represent the cloud masses (Fig. 2), but here we use larger bold points to highlight systems with $m_{2} / m_{1}>0.1$ and $\left(m_{2}+m_{1}\right) / M_{\mathrm{c}}>0.1$, i.e. the observable binary systems. The smaller faint points represent all other systems in the dataset, i.e. the satellite systems and atomistic binaries.

binary inclination (degrees)

$90^{\circ}$

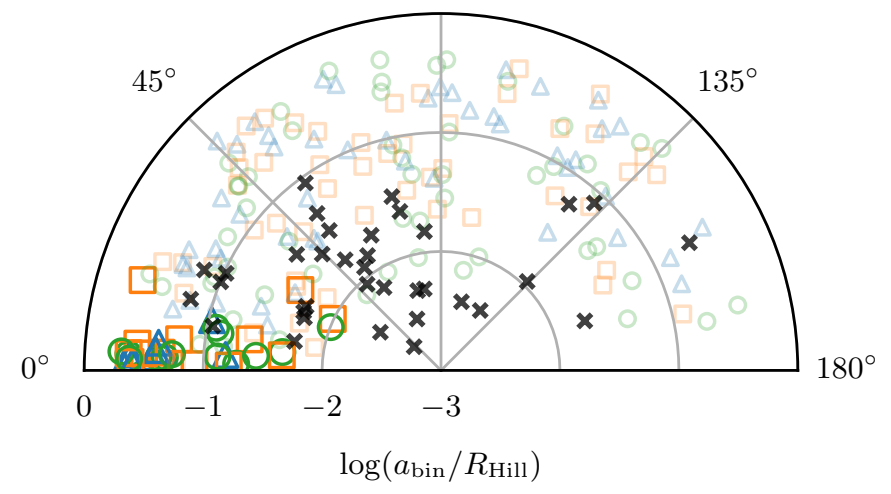

Fig. 9. Binary mutual orbit inclination against semimajor axis relative to the Hill radius, recreated from Fig. 1 of Grundy et al. (2019). We overplot the binary systems from our simulations using the same marker shapes and colours as in Fig. 2 to represent the cloud mass. As in Fig. 8, the larger bold points highlight the observable binary systems and the observed TNBs are black crosses.

have included our simulated binary systems, where $R_{\text {Hill }}$ was calculated at $30 \mathrm{AU}$, which is the formation distance of binaries through gravitational collapse in this study. Gravitational collapse does not reproduce the tightest TNBs, that is, those with $\log \left(a_{\text {bin }} / R_{\text {Hill }}\right)<-2$. This could once more be due to the biases in the simulation caused by particle inflation $f^{*}$ preventing close systems from forming (Sect. 3.3.1). Alternatively, it may be that the systems produced by gravitational collapse evolve onto tighter orbits after formation. As pointed out by 
Nesvorný et al. (2019), Kozai cycles and tidal friction (KCTF), which leads to the tightening and circularisation of orbits (Porter \& Grundy 2012), can be particularly strong for wider binaries above a critical separation $a_{\text {bin }} / R_{\text {Hill }}>0.1$ at inclinations of $i_{\text {bin }} \sim 90^{\circ}$. This could explain what would happen to the excess of wide high-inclination objects in our dataset, compared to the dearth of observed TNBs with such orbits in Fig. 9, over the long period of time after their formation.

In the simulated binary dataset $29 \%$ of systems have retrograde orbits. In comparison, the observed TNBs have $20 \%$ retrograde orbits (Grundy et al. 2019), although we note that the majority of the simulated binaries lie outside the observational limits in Fig. 6. When we consider only the observable binaries, highlighted in Figs. 8 and 9, these systems have a 100\% prograde low-inclination distribution. Assuming they formed through gravitational collapse, the observed TNB inclination distribution is a combination of the inclination of pebble clouds formed by streaming instability and the subsequent $\sim 4 \mathrm{Gyr}$ of Solar System evolution. The simulated binary inclinations are given relative to the $x y$ plane of the Hill frame, which is also the heliocentric orbital plane of the rotating pebble cloud (which we have assumed always rotates about the $\hat{z}$-axis). This means that we have not taken into account that $\sim 20 \%$ of pebble clouds generated by streaming instability rotate retrograde, and so would any binaries (that we would classify as observable) that formed from them (Nesvorný et al. 2019). This is because the findings of Nesvorný et al. (2010) showed that the inclination of binaries produced by gravitational collapse was low and prograde relative to the collapsing cloud. This observation was used by Nesvorný et al. (2019) to directly equate the obliquity of a pebble cloud formed by streaming instability to the inclination of the binary system that is formed by gravitational collapse of that cloud. To directly compare our simulated binary orbits to the observed TNBs in Fig. 9 we would need to apply the streaming instability cloud inclination distribution and the effects of post formation evolution. This would include not only KCTF, but also the collisional and close encounter history, which could alter or destroy the binary orbit after its formation (Nesvorný et al. 2011, 2018a; Parker \& Kavelaars 2010, 2012; Brunini \& Zanardi 2016).

Figure 10 demonstrates how the binary orbital elements scale with the binary mass parameters. There are a large number of high-eccentricity $\left(e_{\text {bin }} \gtrsim 0.5\right)$ and retrograde $\left(i_{\text {bin }}>90^{\circ}\right)$ orbits in our dataset, whereas in Fig. 5 of Nesvorný et al. (2010) there is a preference for lower eccentricities and only prograde systems. In general, these more excited orbits occur for the lower mass binaries $\left(m_{1}+m_{2}\right) / M_{\mathrm{c}} \lesssim 0.1$, that is, these are the additional atomistic binaries detected by the deeper orbit search in this work. Furthermore, at higher system masses the high $e_{\text {bin }}$ and $i_{\text {bin }}$ systems have low mass ratios $m_{2} / m_{1} \lesssim 0.1$, that is, they are the satellite systems where a small companion has been captured around a larger body.

For the complete dataset of 188 simulated binary orbits, $71 \%$ of systems were prograde (median inclination $=32^{\circ}$ ) and $29 \%$ were retrograde $\left(\right.$ median inclination $\left.=123^{\circ}\right)$. In contrast to the observed TNBs of Grundy et al. (2019), we found no obvious trend for wide binaries to have low inclinations, but as mentioned above we have presented a distribution for the time of formation in the rotating reference frame of the pebble cloud undergoing gravitational collapse. When we considered only the simulated binary systems with $m_{2} / m_{1}>0.1$ and $\left(m_{2}+m_{1}\right) / M_{\mathrm{c}}>0.1$ (i.e. the observable binaries), all 35 are prograde, and these systems had a median inclination of $3.5^{\circ}$ (Fig. 9). Furthermore, Figs. 8 and 10 show that the observable binaries generally have only moderate eccentricities. These properties agree with Nesvorný

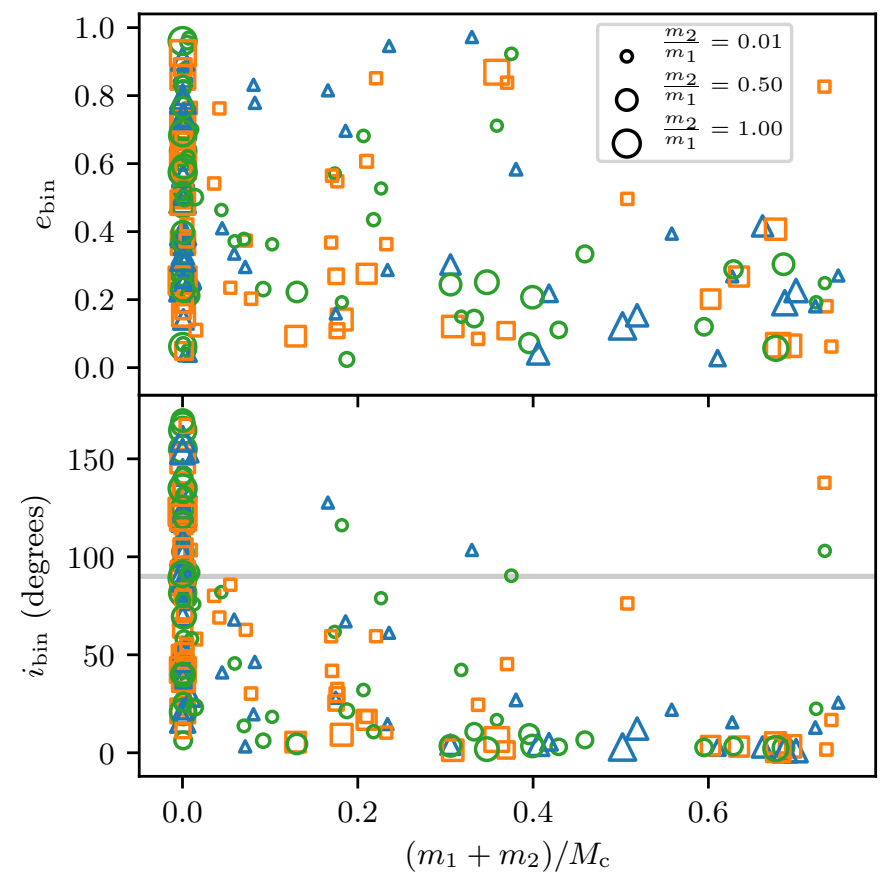

Fig. 10. Relation between the binary orbital parameters (eccentricity and inclination) and the binary mass parameters. The normalised system mass is shown on the $x$-axis, and the mass ratio is represented by marker size. Marker shape and colour denote cloud mass (same as Fig. 2).

et al. (2010, 2019), in which gravitational collapse produces prograde systems with low inclination relative to the rotation of the cloud and $e_{\text {bin }}<0.6$.

\section{Discussion}

\subsection{Origin of contact binaries}

Our results have shown that gravitational collapse is an extremely efficient method of producing binary systems with a range of properties. Stern et al. (2019) noted that gravitational collapse easily forms the nearly equal size ratios of an Arrokoth-like object, with low merger speeds (Nesvorný et al. 2010; Fraser et al. 2017), albeit for higher mass systems (see also Umurhan et al. 2019; McKinnon et al. 2019). The collisional origin of Arrokoth was then investigated further by McKinnon et al. (2020), who showed that a low-velocity impact can indeed preserve the bi-lobate structure. Furthermore, Grishin et al. (2020) demonstrated that dynamical evolution of an initially wide binary can lead to an Arrokoth forming gentle merger. As pointed out by Nesvorný et al. (2018b) for the case of the comet 67P, Simon et al. (2017) provided evidence that streaming instability may scale down to produce less massive $<100 \mathrm{~km}$ objects. High-resolution simulations of the streaming instability by Li et al. (2019) suggest that there could be a turnover in the mass distribution of clouds formed by streaming instability at $\sim 5 \times 10^{17} \mathrm{~kg}$ (Appendix B), implying that clouds with masses lower than this may be less common.

In Fig. 6 we demonstrated that gravitational collapse can produce both large and small binary systems from a single pebble cloud, including those that are equivalent to the masses of the components of Arrokoth. Therefore we do not need to rely entirely on clouds formed by streaming instability scaling all the way down to masses similar to Arrokoth; with gravitational collapse small binaries can be produced in parallel to the larger 


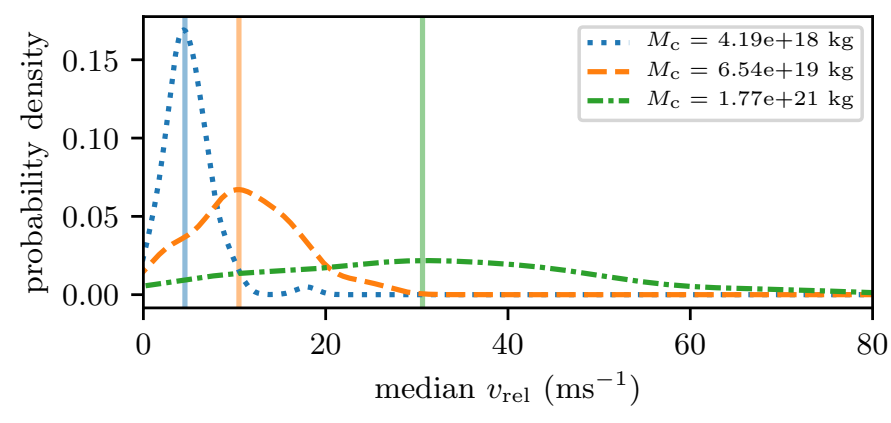

Fig. 11. Probability density distribution of the median collisional velocity for all recorded collisions with $t>50 \mathrm{yr}$ for each gravitationalcollapse simulation. Line style and colour denote cloud mass. The median value of each distribution is shown by a vertical line.

TNBs. It has also been pointed out that Arrokoth has an obliquity close to $90^{\circ}$, and that it would be uncommon to produce such an inclined orbit through gravitational collapse (McKinnon et al. 2019). As shown in Fig. 10, the low-mass binary orbits in our dataset are not constrained to have low prograde binary orbits. Rather, our simulations produce just as many perpendicular orbit binaries as those with low inclinations for systems in the mass range of Arrokoth.

Regardless of the way it formed, a low-mass proto-Arrokoth binary system would have to subsequently evolve into a contact binary. As discussed by Stern et al. (2019), McKinnon et al. (2020) and Grishin et al. (2020), the binary system would have had to lose angular momentum in order for the components to come together. Proposed mechanisms include gas drag, the Kozai-Lidov mechanism, tidal evolution, and collisions. Furthermore, this could possibly be achieved by ejection of another bound body, which is certainly possible given that gravitational collapse commonly produces high-multiplicity systems (Sect. 3.2).

\subsubsection{Formation of contact binaries through gentle collisions}

We also considered the possibility that a contact binary forms directly through the collision of two bodies during cloud collapse (McKinnon et al. 2019, 2020). In gravitational collapse the mean particle velocity in the collapsing cloud depends on cloud mass. In Fig. 11 we present the distribution of median collisional velocities for all collisions recorded in the second half of all simulations $(t>50 \mathrm{yr})$ to gauge what the collisional environment in the cloud is like after the initial cloud collapse and particle growth phase. The median collisional velocities are $\sim 5,10$, and $30 \mathrm{~m} \mathrm{~s}^{-1}$ for the low, intermediate, and high cloud mass, respectively.

If the collisional velocity is low enough then a merging collision may occur, possibly producing a contact binary similar to Arrokoth. As mentioned in Sect. 2.4, merging collisions are possible for $v_{\text {rel }}<v_{\text {esc }}$. The density and mass of Arrokoth is unknown. Using the observed volume, when we assume a characteristic cometary density of $500 \mathrm{~kg} \mathrm{~m}^{-3}$ up to our fiducial $1000 \mathrm{~kg} \mathrm{~m}^{-3}$, then the system mass is $m_{\mathrm{A}}=1-2 \times 10^{15} \mathrm{~kg}$. The escape velocity is therefore $v_{\text {esc }}=\left(2 G m_{\mathrm{A}} / r_{\mathrm{A}}\right)^{1 / 2}=4-6 \mathrm{~m} \mathrm{~s}^{-1}$. For Arrokoth, $v_{\text {esc }}$ is comparable to the median collisional velocity of the lowest cloud mass, although $\sim 17 \%$ of collisions in the intermediate cloud mass also have $v_{\text {rel }} \leq 5 \mathrm{~m} \mathrm{~s}^{-1}$. When we consider only the collisions between particles with masses $m_{1}+m_{2}<m_{\mathrm{A}}$, the median collisional velocities are even lower: $\sim 2$ and $6 \mathrm{~m} \mathrm{~s}^{-1}$ for the low and intermediate cloud masses, respectively. Therefore collisions in these clouds would frequently be merging for Arrokoth-mass particles. The low cloud mass simulations are able to produce binary systems comparable to the mass of Arrokoth (Fig. 6), and therefore $M_{\mathrm{c}} \lesssim$ $4.2 \times 10^{18} \mathrm{~kg}$ pebble clouds appear to be good candidates for producing an Arrokoth-like system through a gentle collisional merger. We note, however, that for the intermediate and high cloud masses, particles as small as Arrokoth are beyond the numerical resolution of our simulations (see Sect. 4.2.2).

The detailed analysis of the collision of the Arrokoth components, for example, deformation of the bodies during collision, is beyond the scope of this paper. However, smoothed particle hydrodynamics studies such as Jutzi \& Asphaug (2015) and Sugiura et al. (2018) indicate that there are impact parameters at these low velocities where collisions can retain the shapes of the colliding particles. Most recently, McKinnon et al. (2020) found that Arrokoth could have formed collisionally provided the impact was no greater than the escape velocity, approximately a few meters per second. Furthermore, if streaming instability and gravitational collapse does indeed scale down to lower cloud masses to form Arrokoth, as implied by Stern et al. (2019), from the trend in Fig. 11 we would expect collisional velocity to also decrease. The collisional environment in these low-mass clouds would then be even more conducive to the formation of a contact binary through a gentle merger.

\subsubsection{Ratio of binary to single planetesimals at low masses}

In Sect. 3.3.4 we discussed the number of bound systems brighter than magnitude 25 compared to singles of the same size that were produced by gravitational collapse. We now consider the number of binaries in the mass range of Arrokoth produced by our simulations, with the assumption that such a binary may evolve into a contact configuration. Figures 2 and 7 show that the distribution of binary system mass with mass ratio and the mass distribution of particles formed by gravitational collapse are similar for each initial cloud mass. By normalising the particle masses with respect to the cloud mass, we see that the overall shape and structure of these distributions is invariant for the cloud masses investigated here. We therefore assumed that these distributions would all scale down to a lower cloud mass of $M_{\mathrm{c} \text {,low }}=5 \times 10^{17} \mathrm{~kg}$, the most common cloud mass produced by the streaming instability simulations of Li et al. (2019). We used our 144 cloud dataset, normalised by the initial cloud mass, to predict the outcome of gravitational collapse for a cloud of mass $M_{\mathrm{c}, \text { low }}$. Using the estimated mass for Arrokoth (Sect. 4.1.1), we considered all binaries within a fractional mass range of $2 \times 10^{-3}-4 \times 10^{-3} M_{\mathrm{c}}$. We found that on average each cloud would produce $\sim 0.07$ such binaries, compared to 0.64 single particles of the same mass. This means that for every Arrokoth-like binary created through the gravitational collapse of a $M_{\text {c,low }}=5 \times 10^{17} \mathrm{~kg}$ mass cloud, we would expect to form about nine single planetesimals in the same mass range.

Because the first cold classical object visited by a spacecraft is a contact binary, this may imply that a large fraction of small cold classicals could be contact binaries. Although we produced a reasonable number of Arrokoth-mass binaries compared to singles, we cannot claim based on our estimate that all cold classical contact binaries started their lives as binaries formed by gravitational collapse. Our predicted binary-to-single ratio is consistent with the estimate of Thirouin \& Sheppard (2019) that $\sim 10-20 \%$ of the cold classical population could be contact binaries. However, this is dependent on all binaries becoming contact systems, and as mentioned previously, we did 
not account for the evolution of the binaries after their formation. In addition, our prediction for the binary-to-single ratio is highly dependent on the choice of $M_{\mathrm{c}, \text { low }}$. We obtained this value from Li et al. (2019), where the conversion from simulation mass units to physical mass units depended on the choice of protoplanetary disc model (Appendix B), let alone any uncertainties in the reported value. For example, when we repeat the above analysis for $M_{\mathrm{c} \text {,low }}=1 \times 10^{17} \mathrm{~kg}$, the binary-to-single ratio is $3: 1$ for Arrokoth masses. This behaviour is a result of the steep powerlaw mass distribution and the break to a flat distribution as shown in Fig. 7. For a given cloud mass, when $m_{\mathrm{A}}$ lies below this break, a surplus of singles will be produced relative to the binaries. An interesting implication of this is that if there is indeed a minimum cloud mass that can form through a streaming instability, and the particle mass distribution is invariant with cloud mass, then there is a critical mass below which nearly all planetesimals form as singles.

\subsection{Simulation limitations}

There are certain limitations to modelling gravitational collapse, as originally discussed by Nesvorný et al. (2010). The use of the particle inflation factor $f^{*}$ is necessary to increase the collision rate in the gravitational-collapse simulation. In reality, the collision rate of the pebble cloud would be high due to the high number density of particles, but when we simulate gravitational collapse we are limited to using a smaller number of computational particles. The use of $f^{*}$ can be problematic. Increasing $f^{*}$ directly increases the collisional cross-sectional area of the particles and therefore affects the rate of collisions and mass accretion. This is shown in Fig. 3, where we found that the types (and frequency) of binaries formed through gravitational collapse were dependent on $f^{*}$. In particular, we found that $f^{*}=30$ was the most efficient producer of binaries with a high system mass, high mass ratio. We followed Nesvorný et al. (2010) in only considering "intermediate" values of $f^{*}$, which strike the balance between no inflation and full inflation that conserves the total surface area of a realistic pebble cloud. Even within this range, $f^{*}$ can be tuned to produce binaries that do (or do not) match observations. Additionally, as shown in Fig. 4, the use of size inflation severely limits the minimum separation of binary systems that can be formed. Tight systems have a tendency to merge into a single planetesimal due to the size of the particles. Furthermore, size inflation means that the particles are unrealistically large and the density is low. Inflated particle density is given by $\rho^{\prime}=\rho / f^{* 3}$, therefore for $f^{*}=100, \rho^{\prime}=\rho \times 10^{-6}$ ! Because of this effect, we were required to shrink particles to their physical radii in order to make a meaningful comparison to the observed systems (see Figs. 5 and 6).

As mentioned in Sect. 2.4, the use of $f^{*}$ can also affect collisional velocities in the cloud. We detected a collision when artificially large inflated particles were overlapping, whereas in reality the particles would have had longer to accelerate towards each other before colliding at their true physical radii. On the other hand, our numerical framework with a finite number of computational particles is biased to have higher collisional velocities than reality in any case. For systems with a fixed total mass, using a smaller number of more massive particles will generally result in stronger gravitational scattering events and therefore higher velocity excitation. All in all, these issues highlight the inherent problems when attempting to simulate a large number of collisionally interacting real particles, and should be borne in mind when interpreting these results.
It has also been assumed that the effects of gas drag are negligible for these binary formation simulations. The presence of gas is required for the initial formation of the cloud through streaming instability, but Nesvorný et al. (2010) showed that the collision timescale of a particle during gravitational collapse dominates the aerodynamic stopping time, therefore the effects of gas can be safely ignored. In addition, they claimed that gas drag should not significantly affect the binary orbits over the timescale of the gas disc. With the current interest in Arrokoth, McKinnon et al. (2019) and Umurhan et al. (2019) stated that this assumption may need to be revisited, especially when the evolution of binary systems into contact binaries is investigated.

\subsubsection{Cloud initial conditions}

The gravitational-collapse simulations may also be limited by the simplicity of the pebble cloud model. We have followed Nesvorný et al. (2010) in using a uniform spherical approximation of the pebble cloud formed by streaming instability. These conditions were originally chosen because the resolution of hydrodynamical simulations of the streaming instability is generally not high enough to resolve the properties of the extremely dense particle clumps. In reality, the streaming instability is a more elaborate process, and the mass, velocity, and angular momentum distributions are more complex. For example, we would expect that mass is generally more concentrated in the centre of the pebble cloud. The streaming instability simulations of Nesvorný et al. (2019) and Li et al. (2019) produced particle clumps with a decreasing radial mass distribution within the Hill sphere of the clump. Furthermore, the shape of the pebble cloud is likely stretched by the local shear forces and would be more ellipsoidal. This increased central density could lead to faster collapse times in the centre of the cloud compared to the outer regions, and an ellipsoidal cloud would have more initial angular momentum, both of which could affect the properties of any binaries formed. Future work that simulates gravitational collapse should consider investigating these more complex initial conditions.

High-resolution hydrodynamic simulations have shown that there are mass limits to the pebble clouds formed by streaming instability. Nesvorný et al. (2019) reported an upper limit to the clouds that formed, and $\mathrm{Li}$ et al. (2019) provided evidence of a turnover at low clump masses (Appendix B). For the high-resolution "Run I" in Li et al. (2019), there is evidence of a turnover in the mass distribution of clumps produced by streaming instability at $5 \times 10^{17} \mathrm{~kg}$, where the distribution peaks. The simulations of Li et al. (2019) and Nesvorný et al. (2019) suggested that the highest mass of a cloud that is produced by streaming instability is $2 \times 10^{20}-1 \times 10^{21} \mathrm{~kg}$. In our simulations, the low and intermediate cloud masses lie between these lower and upper bounds, and we see now that the highest cloud mass appears to be unlikely to form by streaming instability. Our results support an upper limit on cloud mass; we have shown in Sect. 3.3.3 that the high cloud mass produces an overabundance of observable single planetesimals and also binary systems that are not observed. Given the turnover and peak in frequency around $M_{\mathrm{c}}=5 \times 10^{17} \mathrm{~kg}$, it would be wise in future work on gravitational collapse to probe lower cloud masses.

\subsubsection{Numerical resolution}

The number of simulation particles, $N_{\mathrm{p}}=10^{5}$, limited the mass of the smallest particles we could investigate for a particular initial cloud mass. Because of the computational limits for an 
$N$-body simulation with interactions between all particles and accurate collision detection, $N_{\mathrm{p}}=10^{5}$ was the highest resolution that was feasible for this work at the time. This is particularly important when we consider objects on Arrokoth-mass scales. For the low, intermediate, and high cloud mass, a particle of mass $m_{\mathrm{A}}=2 \times 10^{15} \mathrm{~kg}$ would be composed of $n=47,3$, and 0.1 simulation particles (each of mass $m_{0}=M_{\mathrm{c}} / N_{\mathrm{p}}$ ), respectively. It is clearly impossible to form such a system with a high cloud mass simulation, and for the intermediate cloud mass $m_{\mathrm{A}}$ is below the orbit search limit of $4 m_{0}$ (Sect. 3.1). Furthermore, we are most interested in objects that have had a reasonable number of collisions, and have undergone collisional evolution due to the process of gravitational collapse, as these are the objects that are most likely to host a bound system. Therefore, only the low cloud mass simulations are suitable for probing such low masses.

As discussed in Sects. 4.1.1 and 4.2.1, the lower collisional velocities and the distribution of cloud masses formed by streaming instability are good reasons to investigate the gravitational collapse of lower cloud masses. We have shown that the process of binary formation through gravitational collapse is generally invariant with initial cloud mass, for example the mass distribution of particles (Fig. 7), the binary system mass and mass ratio distribution (Fig. 2), and the binary orbital properties (Figs. 9 and 10), therefore we would expect the results for lower cloud masses to be largely the same as well. We would expect such simulations to form a higher number of binaries of mass comparable to Arrokoth (Sect. 4.1.2), and moreover, $m_{0}$ would be a much smaller fraction of $m_{\mathrm{A}}$. Therefore such binaries would be better resolved as they must undergo more collisions to form.

\subsubsection{Completeness of binary identification}

Our results may be limited by our methods of searching for bound particles. Only the $N_{\lim }=100$ most massive particles were searched for orbits, as described in Sect. 3.1, therefore we may have missed a small fraction of bound systems. This cut was made because after gravitational collapse a single particle typically dominated in mass (Fig. 7), which may be a host to bound companions. Our orbit search was designed primarily to find this case, but also any additional bound systems of significant mass (the more massive particles are most likely to host companions). We may miss some systems, especially if they are of low mass, which is particularly relevant for candidate Arrokoth-like systems. When we tested searching a larger number of particles $\left(N_{\lim }=200\right)$ only a small number of additional orbits were found, and we always detected the high system mass and mass ratio systems, as expected. Another issue is that thus far we only considered the orbits found at the end of the simulation, at $t=100 \mathrm{yr}$. However, there could be a number of lower mass binaries that are ejected from the simulation box at earlier times.

Furthermore, we chose to search for bound orbits between pairs of particles, as opposed to assessing the total energy of clumps of particles for binding. This means that we could have missed particles that are not bound to the primary particle, but are bound to the centre of mass of the clump. Any particles that we miss would most likely be only weakly bound as they would either be distant or have a high velocity relative to the primary. We chose our method for its simplicity; structure-finding in $N$-body simulations is a complex problem, and the results may depend on the choice of algorithm (Knebe et al. 2013). The pair-wise orbit search was suitable in our analysis as this method generally identifies high-multiplicity systems where one or more components are only instantaneously bound. During the $10^{4} \mathrm{yr}$ of further dynamical evolution the multiplicity of these systems was

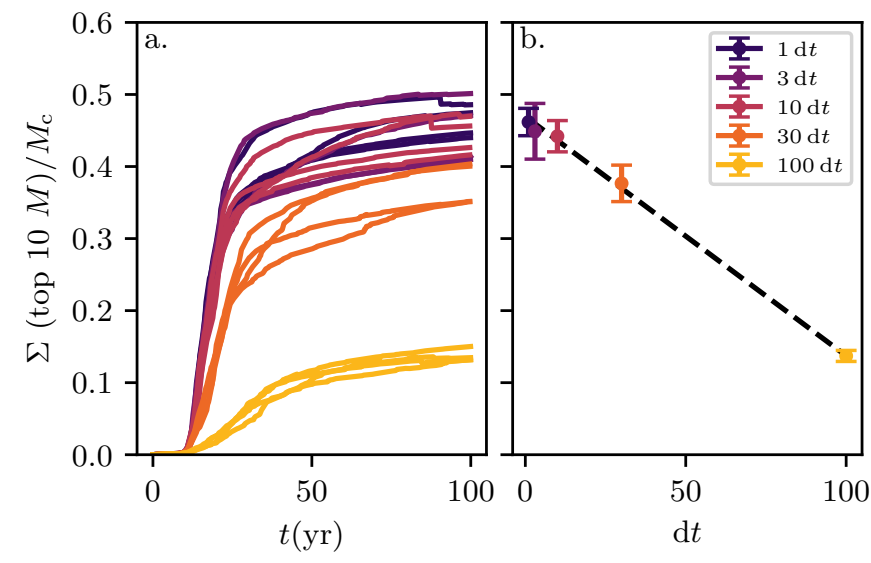

Fig. 12. Effect of changing the timestep on mass accretion in a test set of simulations with parameters $f^{*}=30, M_{\mathrm{c}}=6.54 \times 10^{19} \mathrm{~kg}, X=0.75$, and four unique seed positions. This set of four simulations was repeated with the timestep $\mathrm{d} t$ increased by factors $1,3,10,30$, and 100, as indicated by line and marker colour in both panels. Panel $a$ : total mass of the ten most massive particles in each simulation as a function of time. Panel $b$ : total mass accreted into the ten most massive particles at $t=100 \mathrm{yr}$, where the error bar markers indicate the mean and standard deviation of each set of four simulations. As the timestep is increased, the mass accretion drops in an approximately linear fashion (dashed line).

always drastically reduced, with the majority of systems evolving to simple binary pairs. Therefore we would not expect additional loosely bound particles (that may be detected when the system energy is considered) to survive this further evolution either.

On the other hand, we investigated the types of binaries that are produced by gravitational collapse in more detail than previous work by performing a deeper orbit search. We included all bound systems that were detected by the orbit-search algorithm, whereas Nesvorný et al. (2010) included only the single most massive system produced by each cloud. Furthermore, Fig. 2 showed that in general the detected systems were well above the detection limits of the orbit search. Using this deep orbit search, we found that it is common for each collapsing cloud to produce several bound systems. We ran 144 gravitational-collapse simulations and detected a total of 287 bound systems of particles at $t=100 \mathrm{yr}$. After $10^{4} \mathrm{yr}$ of dynamical evolution we were left with 223 systems; each cloud produced $\sim 1.5$ bound systems on average. Gravitational collapse is therefore an extremely efficient mechanism for producing bound systems of planetesimals.

\subsubsection{Collision detection and mass accretion}

In Sect. 3.3.2 we found that our simulations generally produced more massive binaries than those of Nesvorný et al. (2010). This would imply that particles in our simulations underwent more merging collisions than in previous work. In order to test this, we investigated how the simulation timestep affects the mass accretion of the most massive particles in the cloud. A sample of four cloud-collapse simulations (parameters: $f^{*}=30$, $M_{\mathrm{c}}=6.54 \times 10^{19} \mathrm{~kg}, X=0.75$, and four seed positions) were repeated with the timestep $\mathrm{d} t$ increased by factors of $1,3,10$, 30 , and 100 . We tracked the total mass of the ten most massive particles as a function of simulation time, as shown in Fig. 12a. Considering the rate of mass accretion and comparing this to similar growth tracks of the Nesvorný et al. (2010) simulations (presented in supplementary Fig. 4 of Fraser et al. 2017), we see that in both cases the highest mass is accumulated early in 
the simulation, after which mass accretion levels off. This initial period of rapid runaway growth typically occurs until $t \sim 2 t_{\mathrm{ff}}$. During this time, the rate of mass accretion is dependent on particle mass $m$ such that $\mathrm{d} m / \mathrm{d} t \propto m^{a}$ and $a>1$. After this time, the cloud has begun to reach a post-collapse equilibrium state where collisions are less frequent (e.g. Fig. 1); the rate of mass accretion slows down and particle growth is no longer runaway.

In Fig. 12b we consider the total mass of the ten most massive particles at $t=100 \mathrm{yr}$. It is clear that as the timestep is increased potential collisions are missed, and mass accretion amongst the largest particles is less efficient. The collision criterion (Sect. 2.3) is no longer satisfied. It is therefore possible that the gravitational-collapse simulations of Nesvorný et al. (2010) may have missed some collisions as a result of using a large timestep and straight line extrapolation collision detection (Richardson et al. 2000). As described by Rein \& Tremaine (2011), particle trajectories in Hill's approximation are curved and not straight. Rein \& Tremaine (2011) therefore stated that "it is better to detect collisions approximately along exact trajectories than the reverse". In our simulations, we have been able to do this by conducting more computationally intensive overlapping particle collision detection, and we tested the collision resolution criterion for all simulations, as described in Sect. 2.3.

Interestingly, it would appear that the differing amounts of mass accretion between the two works have not significantly affected the main results. Both our work and that of Nesvorný et al. (2010) successfully produce high-mass ratio binaries that have similar properties to the observed TNBs: low to moderate eccentricity, low prograde inclinations, and separations ranging $\sim 10^{3}-10^{8} \mathrm{~km}$. The only difference is that our binary system masses are systematically more massive than those of Nesvorný et al. (2010), which offers another explanation for why our results favour the collapse of low-mass clouds (Fig. 6).

\section{Conclusions}

We have investigated in detail the formation of transneptunian binaries through the gravitational collapse of a pebble cloud. This is the first independent investigation into this mechanism since it was originally presented by Nesvorný et al. (2010). Our main results are summarised below.

1. Gravitational collapse is extremely efficient at producing bound systems, frequently producing multiple bound systems per cloud.

2. We reproduce the main findings of Nesvorný et al. (2010); gravitational collapse produces binaries with similar mass, wide separation, moderate eccentricity, and low prograde inclination.

3. By performing a deeper orbit search, we found that a range of additional binaries were produced by each cloud; these span a range of system masses, mass ratios, and binary orbital properties.

4. Our results support the upper limit to cloud mass reported in high-resolution streaming instability simulations; the highest cloud mass in our simulations produces binaries that have no observational counterpart, and it is also inconsistent with all planetesimals forming as binaries.

5. Gravitational collapse can produce systems that might evolve into Arrokoth-like objects, with low system mass, lowvelocity merging collisions, and high-obliquity binary orbits.

Our study reproduces and supports some of the results of Nesvorný et al. (2010); gravitational collapse can form binary systems that have nearly equal-size components, prograde lowinclination, and moderate-eccentricity orbits. When compared to the latest TNB data, we find that we can produce binary systems with similar properties to what is observed. In our dataset, observations are best matched by gravitational collapse of a lowmass cloud. Furthermore, by performing a deeper orbit search on particles in the collapsed cloud, we have found that a single cloud can produce more than one bound system. These systems display a diverse range of properties when system masses, mass ratios, binary orbital elements, and multiplicity are considered. We have shown that gravitational collapse is an extremely efficient mechanism for producing bound planetesimal systems.

The collapse of high-mass clouds $\left(M_{\mathrm{c}}=1.77 \times 10^{21} \mathrm{~kg}\right)$ produces large equal-mass binaries for which there is no observational equivalent. This disfavours formation of TNBs through collapse of high-mass clouds and supports the simulations of Nesvorný et al. (2019), which constrain the upper mass of clouds formed by streaming instability. This is reinforced by high-mass clouds producing an excess of single planetesimals. Only the collapse of a low-mass cloud can produce all planetesimals as binaries, a requirement of Fraser et al. (2017). Moreover, when we consider the distribution of binary orbital elements in our dataset (including those that are currently outside the detectable limits), we produce a much wider range of eccentricity and inclination than what is seen in the TNBs observed today. These results could be used in future work to constrain the evolution of binary systems from formation until the present day, testing models of KCTF, collisions, encounters, and migration.

Importantly, by performing a deeper orbit search we find binary systems down to very low masses, typical of objects such as $2014 \mathrm{MU}_{69}$ Arrokoth. We show that a low-mass collapsing cloud has a favourable low-velocity environment for merging collisions that may preserve the shape of components. This lends support to theories of formation of contact binaries from a binary system formed by gravitational collapse, but this requires further investigation into the evolution of TNBs over longer timescales and detailed study into collisions between components, which was recently addressed by McKinnon et al. (2020). In addition, the low-mass binaries in our dataset are not constrained to the same inclination distribution as the systems found by Nesvorný et al. (2010), which perhaps offers a route of forming an extremely oblique Arrokoth.

Acknowledgements. We thank the anonymous referee for their considered review and useful comments which improved this work. J.R. acknowledges funding from the Northern Ireland Department for Education. W.F., A.F. and P.L. acknowledge support from Science and Technology Facilities Council grant ST/P0003094/1. J.R. also thanks the LSSTC Data Science Fellowship Program, which is funded by LSSTC, NSF Cybertraining Grant \#1829740, the Brinson Foundation, and the Moore Foundation; his participation in the program has benefited this work. Simulations in this paper made use of the REBOUND code which is freely available at http://github.com/hannorein/rebound. J.R. is grateful for the assistance of Hanno Rein in developing the code used in this study, through his active support of REBOUND users. J.R. thanks David Nesvorný in particular, for providing valuable insight and guidance throughout the project, as well as Will Grundy for helping to interpret TNB observations. J.R. is also appreciative the support of the Astrophysics Research Centre QUB, in particular Tom Seccull and Richard Smith. This research made extensive use of the HPC facilities of Queen's University Belfast, and also the facilities of the Canadian Astronomy Data Centre operated by the National Research Council of Canada with the support of the Canadian Space Agency. The following software packages were also used in this work: matplotlib (Hunter 2007), numpy (Oliphant 2006; van der Walt et al. 2011), scipy (Virtanen et al. 2020), scikit-learn (Pedregosa et al. 2011) and pandas (McKinney 2010).

\section{References}

Barnes, J., \& Hut, P. 1986, Nature, 324, 446

Benecchi, S. D., Noll, K. S., Grundy, W. M., et al. 2009, Icarus, 200, 292

Benecchi, S. D., Noll, K. S., Grundy, W. M., \& Levison, H. F. 2010, Icarus, 207 978 
Birnstiel, T., Fang, M., \& Johansen, A. 2016, Space Sci. Rev., 205, 41 Brauer, F., Dullemond, C. P., \& Henning, T. 2008, A\&A, 480, 859

Brown, M. 2019, How many dwarf planets are there in the outer solar system? http: //web.gps. caltech. edu/ mbrown/dps.html

Brunini, A., \& Zanardi, M. 2016, MNRAS, 455, 4487

Carrera, D., Gorti, U., Johansen, A., \& Davies, M. B. 2017, ApJ, 839, 16

Chiang, E., \& Youdin, A. N. 2010, Ann. Rev. Earth Planet. Sci., 38, 493

Ester, M., Kriegel, H.-P., Sander, J., \& Xu, X. 1996, in Proceedings of the Second International Conference on Knowledge Discovery and Data Mining, KDD'96 (AAAI Press), 226

Fraser, W. C., Brown, M. E., Morbidelli, A. r., Parker, A., \& Batygin, K. 2014, ApJ, 782, 100

Fraser, W. C., Bannister, M. T., Pike, R. E., et al. 2017, Nat. Astron., 1, 0088

Funato, Y., Makino, J., Hut, P., Kokubo, E., \& Kinoshita, D. 2004, Nature, 427, 518

Gladman, B., Marsden, B. G., \& Vanlaerhoven, C. 2008, The Solar System beyond Neptune, eds. M. A. Barucci, H. Boehnhardt, D. P. Cruikshank, A. Morbidelli, \& R. Dotson (Tucson, AZ: University of Arizona Press), 43

Goldreich, P., Lithwick, Y., \& Sari, R. 2002, Nature, 420, 643

Grishin, E., Malamud, U., Perets, H. B., Wandel, O., \& Schäfer, C. M. 2020, Nature, 580, 463

Grundy, W. 2019, Mutual Orbits of Binary Transneptunian Objects, http:// www2 . lowell . edu/users/grundy/tnbs/, accessed: 2 Dec 2019

Grundy, W. M., Noll, K. S., Nimmo, F., et al. 2011, Icarus, 213, 678

Grundy, W. M., Noll, K. S., Roe, H. G., et al. 2019, Icarus, 334, 62

Güttler, C., Blum, J., Zsom, A., Ormel, C. W., \& Dullemond, C. P. 2010, A\&A 513, A56

Hagberg, A. A., Schult, D. A., \& Swart, P. J. 2008, in Proceedings of the 7th Python in Science Conference, eds. G. Varoquaux, T. Vaught, \& J. Millman, Pasadena, CA USA, 11

Hunter, J. D. 2007, Comput. Sci. Eng., 9, 90

Johansen, A., Oishi, J. S., Mac Low, M.-M., et al. 2007, Nature, 448 , 1022

Johansen, A., Youdin, A., \& Mac Low, M.-M. 2009, ApJ, 704, L75

Johansen, A., Mac Low, M.-M., Lacerda, P., \& Bizzarro, M. 2015, Sci. Adv., 1, 1500109

Jutzi, M., \& Asphaug, E. 2015, Science, 348, 1355

Knebe, A., Pearce, F. R., Lux, H., et al. 2013, MNRAS, 435, 1618

Kokubo, E., \& Ida, S. 1996, Icarus, 123, 180

Lacerda, P. 2011, AJ, 142, 90

Lacerda, P., Fornasier, S., Lellouch, E., et al. 2014, ApJ, 793, L2

Leinhardt, Z. M., \& Stewart, S. T. 2012, ApJ, 745, 79

Li, R., Youdin, A. N., \& Simon, J. B. 2019, ApJ, 885, 69

Marsset, M., Fraser, W. C., Bannister, M. T., et al. 2020, Planet. Sci. J., 1, 16

McKinney, W. 2010, in Proceedings of the 9th Python in Science Conference, eds. S. van der Walt, \& J. Millman, 5

McKinnon, W. B., Stern, S. A., Weaver, H. A., et al. 2019, Lunar and Planetary Science Conference, 2767

McKinnon, W. B., Richardson, D. C., Marohnic, J. C., et al. 2020, Science, 367, aay6620

Morbidelli, A., \& Nesvorny, D. 2020 The Trans-Neptunian Solar System, eds. D. Prialnik, M. A. Barucci, \& L. Young (Amsterdam: Elsevier), 25
Morbidelli, A., Levison, H. F., \& Gomes, R. 2008, The Solar System Beyond Neptune, eds. M. A. Barucci, H. Boehnhardt, D. P. Cruikshank, A. Morbidelli, \& R. Dotson (Tucson, AZ: University of Arizona Press), 275

Mustill, A. J., Davies, M. B., \& Johansen, A. 2018, MNRAS, 478, 2896

Nakazawa, K., \& Ida, S. 1988, Prog. Theor. Phys. Suppl., 96, 167

Nesvorný, D. 2008, AAS/Division Planet. Sci. Meeting Abstracts, 40, 38.02

Nesvorný, D., Youdin, A. N., \& Richardson, D. C. 2010, AJ, 140, 785

Nesvorný, D., Vokrouhlický, D., Bottke, W. F., Noll, K., \& Levison, H. F. 2011, AJ, 141, 159

Nesvorný, D., Vokrouhlický, D., Bottke, W. F., \& Levison, H. F. 2018a, Nat. Astron., 2, 878

Nesvorný, D., Parker, J., \& Vokrouhlický, D. 2018b, AJ, 155, 246

Nesvorný, D., Li, R., Youdin, A. N., Simon, J. B., \& Grundy, W. M. 2019, Nat. Astron., 3, 808

Noll, K. S., Grundy, W. M., Chiang, E. I., Margot, J. L., \& Kern, S. D. 2008, The Solar System Beyond Neptune, eds. M. A. Barucci, H. Boehnhardt, D. P. Cruikshank, A. Morbidelli, \& R. Dotson (Tucson, AZ: University of Arizona Press), 345

Oliphant, T. 2006, NumPy: A guide to NumPy (USA: Trelgol Publishing)

Parker, A. H., \& Kavelaars, J. J. 2010, ApJ, 722, L204

Parker, A. H., \& Kavelaars, J. J. 2012, ApJ, 744, 139

Parker, A. H., Kavelaars, J. J., Petit, J.-M., et al. 2011, ApJ, 743, 1

Pedregosa, F., Varoquaux, G., Gramfort, A., et al. 2011, J. Mach. Learn. Res., 12, 2825

Petit, J. M., Kavelaars, J. J., Gladman, B., \& Loredo, T. 2008, Size Distribution of Multikilometer Transneptunian Objects, ed. M. A. Barucci, H. Boehnhardt, D. P. Cruikshank, A. Morbidelli, \& R. Dotson, 71

Porter, S. B., \& Grundy, W. M. 2012, Icarus, 220, 947

Rein, H., \& Liu, S. F. 2012, A\&A, 537, A128

Rein, H., \& Tremaine, S. 2011, MNRAS, 415, 3168

Richardson, D. C., Quinn, T., Stadel, J., \& Lake, G. 2000, Icarus, 143, 45

Sheppard, S. S., \& Jewitt, D. 2004, AJ, 127, 3023

Simon, J. B., Armitage, P. J., Li, R., \& Youdin, A. N. 2016, ApJ, 822, 55

Simon, J. B., Armitage, P. J., Youdin, A. N., \& Li, R. 2017, ApJ, 847, L12

Stephens, D. C., \& Noll, K. S. 2006, AJ, 131, 1142

Stern, S. A., Weaver, H. A., Spencer, J. R., et al. 2019, Science, 364, aaw9771

Sugiura, K., Kobayashi, H., \& Inutsuka, S. 2018, A\&A, 620, A167

Thirouin, A., \& Sheppard, S. S. 2019, AJ, 157, 228

Umurhan, O. M., Kavelaars, J. J., Cuzzi, J. N., et al. 2019, Lunar and Planetary Science Conference, 2809

van der Walt, S., Colbert, S. C., \& Varoquaux, G. 2011, Comput. Sci. Eng., 13, 22

Virtanen, P., Gommers, R., Oliphant, T. E., et al. 2020, Nat. Methods, 17, 261

Walsh, K. J., \& Jacobson, S. A. 2015, Asteroids IV (Tucson, AZ: University of Arizona Press), 375

Weidenschilling, S. J. 1977, MNRAS, 180, 57

Weidenschilling, S. J. 2002, Icarus, 160, 212

Willmer, C. N. A. 2018, ApJS, 236, 47

Yang, C. C., Johansen, A., \& Carrera, D. 2017, A\&A, 606, A80

Youdin, A. N., \& Goodman, J. 2005, ApJ, 620, 459 


\section{Appendix A: Calculating magnitudes}

We calculate an apparent magnitude (in waveband $\lambda$ ) of an object of radius $r \mathrm{~km}$ and geometric albedo $p_{\lambda}$ using the equations in Petit et al. (2008) and Noll et al. (2008),

$$
\begin{aligned}
\mathrm{m}_{\lambda} & =\mathrm{m}_{\odot, \lambda}-2.5 \log \left(\frac{p_{\lambda} r^{2} \phi(\alpha)}{2.25 \times 10^{16} R^{2} \Delta^{2}}\right) \\
& =5 \log \left(\sqrt{2.25 \times 10^{16}} 10^{0.2 \mathrm{~m}_{\odot, \lambda}} \frac{R \Delta}{\sqrt{p_{\lambda}} r}\right) \\
& =5 \log \left(C_{\lambda} \frac{R \Delta}{\sqrt{p_{\lambda}} r}\right),
\end{aligned}
$$

where for the $V$ band, $\mathrm{m}_{\odot, V}=-26.76$ (Willmer 2018) is the $V$ -band solar magnitude, $C_{V}=664.5 \mathrm{~km}$ (Noll et al. 2008), and we have assumed that the phase function $\phi=1$ (i.e. phase angle $\alpha=0$ ) and the object has no rotational variation. $R$ is the heliocentric distance of the object and $\Delta=R-1$ is the geocentric distance for $\alpha=0$, both in AU.

The magnitude difference between a binary with components of size $r_{1}$ and $r_{2}$ is then

$\Delta \mathrm{m}_{\lambda}=\mathrm{m}_{\lambda, 2}-\mathrm{m}_{\lambda, 1}=5 \log \left(\frac{r_{1}}{r_{2}} \sqrt{\frac{p_{\lambda, 1}}{p_{\lambda, 2}}}\right)$.

\section{Appendix B: Streaming instability masses}

Nesvorný et al. (2019) reported a mass distribution of clouds formed by streaming instability. In their supplementary information, they discussed the highest planetesimal mass that can be produced by streaming instability and the conversion between simulation mass units and physical mass units, which requires a disc temperature. The maximum cloud mass produced by their runs $\mathrm{A}$ and $\mathrm{C}$ is

$M_{\max } \sim 0.25-0.75 T_{25}^{3 / 2} M_{\text {Ceres }}$,

where $M_{\text {Ceres }}=9.4 \times 10^{20} \mathrm{~kg}$ is the mass of Ceres and $T_{25}=T /(25 \mathrm{~K})$ for disc temperature $T$. A fully flared disc model predicts a temperature profile of exponent $-3 / 7$ (Chiang \& Youdin 2010). Therefore Nesvorný et al. (2019) used

$T=39 R_{45}^{-3 / 7}$,

where $R_{45}=r /(45 \mathrm{AU})$ and $r$ is the radial heliocentric distance.
Using these relations, for $r=45 \mathrm{AU}, T=39 \mathrm{~K}, M_{\max } \sim$ $4.6 \times 10^{20}-1.4 \times 10^{21} \mathrm{~kg}$. For $r=30 \mathrm{AU}, T=46 \mathrm{~K}, M_{\max } \sim$ $5.9 \times 10^{20}-1.8 \times 10^{21} \mathrm{~kg}$. Therefore we assume a maximum cloud mass of $\sim 1 \times 10^{21} \mathrm{~kg}$.

In their high-resolution streaming instability simulations, Li et al. (2019) found evidence for a turnover at low mass in the distribution of cloud masses produced by streaming instability. They analysed two simulations, Run I and Run II (equivalent to the Nesvorný et al. 2019 runs A and C respectively). Both have the following dimensionless simulation parameters: global radial pressure gradient parameter $\Pi=0.05$, and relative strength of particle self-gravity to the tidal shear $\tilde{G}=0.05$. Run I has a stopping time and gas-to-surface density $\left(\tau_{s}, Z\right)=(2.0,0.1)$ and is higher resolution $(\Delta x=H / 5120)$ in a smaller domain. Run II has $\left(\tau_{s}, Z\right)=(0.3,0.02)$ and is lower resolution (half of Run I) over a larger domain. The dimensionless simulation mass units for the runs correspond to physical masses $M_{G}=0.19$ and $0.0015 M_{\text {Ceres }}$, respectively. As for Nesvorný et al. (2019) above, these values are dependent on disc conditions, such as disc radius $R$ and temperature profile $T \propto R^{-3 / 7}$.

The high resolution of Run I allows the production of lower cloud masses than Run II, thus allowing investigation of the low end of the cloud mass distribution. Figure 4 of Li et al. (2019) provides evidence of a turnover in the mass distribution below a peak at $\sim 0.003 M_{G}=5.7 \times 10^{-4} M_{\text {Ceres }}=5.4 \times 10^{17} \mathrm{~kg}$. These results imply that clouds of mass $5.4 \times 10^{17} \mathrm{~kg}$ should be the most common clouds produced by streaming instability.

\section{Appendix C: Supplementary animation}

We include an animation showing the complete process of gravitational collapse ${ }^{3}$ for a cloud with the parameters $f^{*}=30$, $X=0.5$ and $M_{\mathrm{c}}=6.54 \times 10^{19} \mathrm{~kg}$. The left-hand panel shows the $x y$ projection of the particle position in the simulation box. The marker size scales linearly with particle radius $r_{f}$, and the colour scales logarithmically with particle mass $m$ according to $m_{\text {rel }}=\log \left(m / m_{0}\right) / \log \left(M_{\mathrm{c}} / m_{0}\right)$. The panel is initially zoomed in on the cloud and then zooms out until it shows the extent of the whole simulation box. The centre of mass of the cloud is marked with a black cross and the position of the most massive particle is shown with a red plus. The right-hand panel shows an expanded view centred on the most massive particle at that timestep. Here the marker size scales with $r_{f}^{2}$ to emphasise the larger particles. The blue ellipse indicates the binary orbit that is detected between the most massive particle and its largest companion. 